\title{
Non-parametric Morphologies of Mergers in the Illustris Simulation
}

\author{
L. A. Bignone ${ }^{1 \star}$, P. B. Tissera ${ }^{2,3}$, E. Sillero ${ }^{4}$, S. E. Pedrosa ${ }^{1}$, L. J. Pellizza ${ }^{5}$ \\ and D. García Lambas ${ }^{4}$ \\ ${ }^{1}$ Instituto de Astronomía y Física del Espacio (IAFE, CONICET-UBA), C.C. 67 Suc. 28, C1428ZAA Ciudad de Buenos Aires, Argentina. \\ ${ }^{2}$ Departamento de Ciencias Físicas, Universidad Andres Bello, Av. Republica 220, Santiago, Chile. \\ ${ }^{3}$ Millennium Institute of Astrophysics, Av. Republica 220, Santiago, Chile \\ ${ }^{4}$ Instituto de Astronomia Teórica y Experimental (CONICET-UNC), Laprida 925, Cordoba, Argentina. \\ ${ }^{5}$ Instituto Argentino de Radioastronomía, CONICET, Camino Gral. Belgrano km 40, Berazategui, Prov. de Buenos Aires, Argentina
}

Accepted XXX. Received YYY; in original form ZZZ

\begin{abstract}
We study non-parametric morphologies of mergers events in a cosmological context, using the Illustris project. We produce mock g-band images comparable to observational surveys from the publicly available Illustris simulation idealized mock images at $z=0$. We then measure non parametric indicators: asymmetry, Gini, $M_{20}$, clumpiness and concentration for a set of galaxies with $M_{*}>10^{10} \mathrm{M}_{\odot}$. We correlate these automatic statistics with the recent merger history of galaxies and with the presence of close companions. Our main contribution is to assess in a cosmological framework, the empirically derived non-parametric demarcation line and average time-scales used to determine the merger rate observationally. We found that 98 per cent of galaxies above the demarcation line have a close companion or have experienced a recent merger event. On average, merger signatures obtained from the $G-M_{20}$ criteria anticorrelate clearly with the elapsing time to the last merger event. We also find that the asymmetry correlates with galaxy pair separation and relative velocity, exhibiting the larger enhancements for those systems with pair separations $d<50 \mathrm{~h}^{-1} \mathrm{kpc}$ and relative velocities $V<350 \mathrm{~km} \mathrm{~s}^{-1}$. We find that the $G-M_{20}$ is most sensitive to recent mergers $(\sim 0.14 \mathrm{Gyr})$ and to ongoing mergers with stellar mass ratios greater than 0.1. For this indicator, we compute a merger average observability time-scale of $\sim 0.2 \mathrm{Gyr}$, in agreement with previous results and demonstrate that the morphologically derived merger rate recovers the intrinsic total merger rate of the simulation and the merger rate as a function of stellar mass.
\end{abstract}

Key words:

cosmology: galaxy formation - galaxies: interactions - galaxies: structure - galaxies: evolution - methods: numerical

\section{INTRODUCTION}

Galaxy mergers are of fundamental importance in the formation and evolution of galaxies, especially in the $\Lambda$ Cold Dark Matter $(\Lambda \mathrm{CDM})$ cosmology where structure grows hierarchically (e.g. White \& Rees 1978). Mergers have an important effect in the mass assembly of galaxies (Guo \& White 2008; Genel et al. 2009), the star formation history (Mihos \& Hernquist 1996; Somerville et al. 2001), the establishment of galaxy morphologies, internal

* E-mail:lbignone@iafe.uba.ar structures (Mihos \& Hernquist 1994; Johnston et al. 1996; Naab \& Burkert 2003; Bell et al. 2008) and the growth and accretion of gas by supermassive black holes (Hopkins et al. 2006). Understanding the role of mergers in the formation of galaxies and their relative importance in comparison to other, more continuous processes, such as cold gas and dark matter accretion is a key challenge for galaxy formation models.

A first step to study the role of mergers in galaxy evolution is the estimation of the merger rate by counting the observed number of events. There are several approaches for the identification of such systems. Galaxy 
pairs with close projected angular separations and low line-of-sight relative radial velocities, for example, can be considered suitable merger candidates (Barton et al. 2000; Patton et al. 2002; Lambas et al. 2003; Lin et al. 2004; De Propris et al. 2005). An alternatively method is the identification of morphologically disturbed galaxies, either through visual inspection (Kampczyk et al. 2007; Bundy et al. 2005; Brinchmann et al. 1998) or by quantitative measurements of non-parametric morphological statistics such as the Gini coefficient $(G)$, the second-order moment of the brightest 20 per cent of the light $\left(M_{20}\right.$, Lotz et al. 2004, hereafter LPM04) and the CAS system formed by the combination of concentration $(C)$ (Wu 1999; Bershady et al. 2000; Conselice et al. 2000), rotational asymmetry $(A)$ (Abraham et al. 1996; Conselice et al. 2000), and clumpiness $(S)$ (Isserstedt \& Schindler 1986; Takamiya 1999; Conselice 2003).

Different methods of merger candidate selection might be sensitive to different merger stages. For example, selecting close pairs imply a higher probability of capturing galaxies in the pre and early merger stages, while morphological disturbances methods are more sensitive to pre, ongoing and post merger stages (Lotz et al. 2011). It is not surprising then, that observational constraints of the merger fractions can differ by up to an order of magnitude and yield very different redshift evolution depending on the method adopted (e.g. Lotz et al. 2008b; de Ravel et al. 2009; Bundy et al. 2009; López-Sanjuan et al. 2009; Conselice et al. 2009).

In order to properly obtain merger rates from observed merger fractions, an observability time-scale (i.e. the average time during which a merging system would be identified as such by applying certain criteria) has to be adopted. This time-scale might be sensitive to a wide variety of factors such as the merger selection criteria, the interacting galaxy properties (e.g. mass ratio, gas fraction, orbital parameters) and observational parameters (e.g. viewing angle, resolution, observed wavelength, S/N ratio). Lotz et al. (2008a) used a series of numerical simulations of equal-mass interacting galaxy pairs to constraint the observability time-scales for a variety of non-parametric morphological statistics. The simulations were processed trough a radiative transfer code that resulted in realistic mock images of the interacting galaxies at different merger stages. Then the images were used to study the dependence of morphological statistics on the merger stage, viewing angle, orbital parameters and gas properties. Similar methods were used to study the effect of merger mass ratio (Lotz et al. 2010a) and gas fraction (Lotz et al. 2010b).

However, the use of isolated merger simulations to derive the observability time-scale comes with a significant limitation, they do not account for the cosmological context of galaxy formation. Instead, the observability time-scales for each merger parameter set (e.g. mass ratios, gas fractions) has to be weighted by the probability distribution of such parameters. Typically, these distributions are poorly constraint observationally and have to be derived from independent, cosmological-scale simulations. Using this methodology, Lotz et al. (2011) successfully reconciled the uneven observational merger rates at $z<1.5$, and were able to differentiate the rates for major and minor mergers. They also compared the evolution of the merger rates with theoretical predictions of galaxy evolution models, finding an excellent agreement for the major merger rate. Conversely, the total merger rate (minor and majors combined) derived from the $G-M_{20}$ diagnostic, was an order of magnitude higher that the rate predicted by the cosmological simulations which were used to derive the distributions of merger parameters. This result suggested a possible underestimation of the $G-M_{20}$ observability time-scale derived from isolated interacting pair simulations (Lotz et al. 2008a, 2010a).

An appealing alternative is the study of the nonparametric morphology indicators of mergers directly selected from cosmological hydrodynamical simulations. In this simulated cosmological context, interacting galaxies cover a wide range of stellar masses, gas fraction, environments, mass ratios and orbital parameters that closely resemble what observational studies of such systems must encounter. Recently Snyder et al. (2015a) used a set of 22 zoomed-in galaxies to quantify the morphological evolution at $z>1$, including the morphological effects of mergers. While zoomed-in simulations certainly capture the cosmological context of galaxy formation, they still constitute a small sample size that explores a limited parameter space. A solution to this limitation is to explore non-parametric morphological statistics of a galaxy catalogue selected from a large simulated volume. This clearly represents a significant technical challenge which only recently has became possible to tackle. At $z=0$, Snyder et al. (2015b) studied the Gini$M_{20}$ morphology of 10808 galaxies from the Illustris simulation (Vogelsberger et al. 2014). These authors found that the morphological distribution of simulated galaxies agreed well with observations, and that important relationships such as the connection between morphological type and stellar mass $\left(\mathrm{M}_{*}\right)$ and morphological type and star formation rate, follow the trends reported by different galaxy surveys.

It is also important to point out that hydrodynamical simulations face important challenges in reproducing the complicated physical processes involved in galaxy formation, and that further improvements in the modelling of certain aspects which regulate star formation such as cooling rates, gas inflows and outflows and feedback should be expected. In the case of the Illustris project, Sparre et al. (2015) found that the observed relationship between star formation rate and stellar mass at $z=0$ and 4 are well reproduced, but not at intermediate redshifts where the normalization of the relationship is too low. Numerical resolution is also an important factor to consider. Sparre et al. (2015) found a paucity of strong starbursts in the Illustris simulation which can affect the appearance of mergers where induced star formation is expected. In fact, this resolution effect was further studied by Sparre \& Springel (2016) where zoom-in simulations of major mergers at 10-40 times higher mass resolution than Illustris where more successful at generating starbursts using the same physics model. Numerical resolution can also impact the mock images generated from cosmological simulations given that the mass of simulated stellar particles can be 2-3 orders of magnitude higher than actual star forming regions, this can affect the galaxy appearance (Torrey et al. 2015) and also their colours and luminosities (Trayford et al. 2015). These caveats affect all currently large volume simulations of this kind since it is not yet possible to simulate such large volumes at higher resolutions.

In this work we take full advantage of the large cosmological volume of the Illustris simulation to derive a statis- 
tically significant number of non-parametric morphological indicators of galaxies subject to diverse environmental situations, including isolated system, merging and interacting pairs. From the publicly available mock images of the Illustris simulation (Torrey et al. 2015) we select a sample of galaxies with $M_{*}>10^{10} \mathrm{M}_{\odot}$ at $z=0$ to study the ability of Gini, $M_{20}$, concentration, clumpiness and asymmetry to successfully classify close pairs, minor mergers and major mergers. We analyse the effectiveness of the empirically derived $G-M_{20}$ and $C A S$ merger diagnostics to distinguish between normal and interacting galaxies in the simulation. Finally, we attempt to reconcile the intrinsic merger rate of the simulation with the merger rate derived using the same techniques often used in observational studies (e.g Lotz et al. 2008b, 2011).

The paper is organized as follows. In section 2 we briefly describe the Illustris simulation and the galaxy samples selected for our analysis. In section 3 we explain the procedure applied to the mock images. In Section 4 we measure the effectiveness of the merger diagnostics in selecting different populations of interacting galaxies or recent merger events. In section 5 we explore the merger rate of the simulation in the light of non-parametric morphological studies. Finally, in section 6 we discuss our results a present our conclusions.

\section{SIMULATED GALAXY SAMPLES}

In the following sections we provide a brief description of the Illustris simulations, the galaxy catalogues and the mock images. We also include a description of the galaxy subsamples defined for our analysis.

\subsection{Overview of the Illustris Simulation}

The Illustris project (Vogelsberger et al. 2014; Genel et al. 2014) consists of a set of large-scale hydrodynamical cosmological simulations with periodic box $106.5 \mathrm{Mpc}$ a side, run with the quasi-Lagrangian AREPO code (Springel 2010). The galaxy formation model (Vogelsberger et al. 2013) includes gas cooling and photo-ionization, star formation and ISM models, stellar evolution (gas recycling and chemical enrichment), stellar supernova feedback and supermassive black holes with quasar-mode and radio-mode feedback (Sijacki et al. 2007, 2015).

The main simulation of the project, Illustris-1 (hereafter, I-1), initially has $1820^{3}$ gas cells and $1820^{3}$ dark matter (DM) particles. The initial mass of gas elements is $1.26 \times 10^{6} \mathrm{M}_{\odot}$, while for $\mathrm{DM}$ particles the mass is $6.26 \times 10^{6} \mathrm{M}_{\odot}$. The I-1 simulation follows structure and galaxy formation across 136 snapshots, culminating at $z=0$ and has been shown to reproduce many of the key observed trends in the local Universe Vogelsberger et al. (2014), with some discrepancies related to the stellar ages of low mass $\left(M_{*} \lesssim 10^{10.5} \mathrm{M}_{\odot}\right)$ galaxies and the quenching of massive galaxies.

The Illustris project adopted the following set of cosmological parameters: $\Omega_{m}=0.2726, \Omega_{\Lambda}=0.7274, \Omega_{b}=0.0456$, $\sigma_{8}=0.809, n_{s}=0.963$ and $h=0.704$, which are consistent with the Wilkinson Microwave Anisotropy Probe (WMAP)-9 measurements (Hinshaw et al. 2013).

\subsection{Galaxy catalogue and mergers trees}

In the I-1 simulation, DM halos were identified using the standard friends-of-friends (FoF) algorithm (Davis et al. 1985) with linking length of 0.2 times the mean particle separation and a minimum number of $32 \mathrm{DM}$ particles. Baryonic elements were assigned to the FoF group of the closest DM particle. Gravitationally bound substructures within the FoF groups were identified using the SUBFIND algorithm (Springel et al. 2001; Dolag et al. 2009) resulting in $4,366,546$ individual subhalos at $z=0$. Subhalos with $\mathrm{M}_{*} \simeq 10^{10} \mathrm{M}_{\odot}$ have approximately 30,000 gas cells, 40, 000 DM particles, and 10,000 star particles. We point out that stellar masses used in this paper are those obtained from the SUBFIND catalogue, without considering truncation at any radius.

From the halo and subhalo group catalogues, Rodriguez-Gomez et al. (2015) constructed the corresponding merger trees using the newly developed SubLink code. They argued that in order to avoid problems caused by the way halo finders distribute mass between substructures, a robust estimation of the mass ratio of galaxies in a merger event can be obtained by taking the two progenitor masses at the moment when the secondary progenitor reaches its maximum stellar mass. Throughout the rest of this paper, we refer to merger events taken from the SuBLink merger trees. Their mass ratios are computed according to the above definition.

\subsection{Mock observations}

Torrey et al. (2015) present a method to generate synthetic images and integrated spectra for galaxies in the Illustris project. They employed the radiative transfer code SUNRISE (Jonsson 2006; Jonsson et al. 2010) to assign a full spectral energy distribution (SED) to each star particle and to generate images of arbitrary field-of-view (FOV) and pixel size for different camera orientations with respect to a galaxy. The SEDs were calculated by assuming the single-age stellar populations models by STARBURST99 (Leitherer et al. 1999, 2010). A simplified empirical dust model of Charlot \& Fall (2000) was also adopted.

The Illustris project made available through its online database ${ }^{1} 6978$ mock observations for galaxies with $M_{*}>10^{10} \mathrm{M}_{\odot}$ at $z=0$. Each galaxy was imaged with four ( $256 \times 256$ pixels) cameras orientated in four different viewing angles which were randomly aligned with respect to the rotational axis of the galaxy. Each camera was placed $50 \mathrm{Mpc}$ away from the galaxy centre and the FOV was set to 10 times the stellar half mass radius for the galaxy. It is important to point out that the mock observations include not only light from the chosen subhalo, but also from all other structures belonging to the same halo that fall within the FOV. Because non-parametric indicators can be affected by the light from projected close companions, this kind of images are specially suitable to perform the kind of morphological perturbation studies we propose here.

These images constitute idealized observations because they do not include noise, camera point-spread-function

1 http://www.illustris-project.org/data/ 
(PSF) blurring or contamination from foreground or background sources. They are meant to be used as a base to compare with observations from different telescopes. Hence, the images must be degraded according to the particular characteristics of the observations to be used to confront them. In this paper, we focus on mock rest-frame g-band morphologies that can be compared to optical and observed morphologies from the ground. In section 3, we describe how the mock $\mathrm{g}$ observations are degraded in order to approximately match real observations.

Our mock image sample has been cleaned of an artefact produced by the image generation algorithm which resulted in some empty images. The issue occurred whenever the halo centre position and the subhalo position were a periodic boundary away from one another (private communication). We found and removed 99 such subhalos resulting in a final sample of 6879 galaxies.

\subsection{Galaxy samples}

From the SubLink merger trees, we define a subsample of major merger remnants made up by galaxies at $z=0$ that experienced at least one major merger in the previous 2 Gyr. We consider a merger as major when the stellar mass ratio $\left(\mu_{*}\right)$ between the secondary and principal components is larger than 0.25 . We also define a subsample of minor merger remnants composed by galaxies at $z=0$ that experienced at least one minor merger in the last 2 Gyr and no major merger. We consider a merger as minor when $\mu_{*}<0.25$. Subhalos included in the merger trees have at least 20 resolution elements between gas and stars (Rodriguez-Gomez et al. 2015) which gives a minimum mass of about $2.6 \times 10^{7} \mathrm{M}_{\odot}$ and a minimum $\mu_{*} \sim 0.001$ for $10^{10}$ $\mathrm{M}_{\odot}$ descendants.. The total major and minor merger remnants subsamples have 322 and 3784 individual galaxies, respectively.

We also define a subsample of 753 close galaxy pairs. We limit the sample to those galaxies having a companion with a stellar mass greater than $10^{8} h^{-1} \mathrm{M}_{\odot}$ at a distance $d \leq 20 \mathrm{~h}^{-1} \mathrm{kpc}$. At these close distances, galaxies are more likely to constitute an on-going merger or to experience disturbed morphologies due to gravitational interaction (e.g. Lambas et al. 2003; Perez et al. 2006). However, not all of these pairs actually constitute mergers. In order to better constrain the on-going merger sample, we also consider the relative velocities between the members of the pairs.

Finally, we define a subsample of 5090 distant pairs formed by galaxies having a companion with a stellar mass greater than $10^{8} h^{-1} \mathrm{M}_{\odot}$ within the range $20<d \leq 100$ $\mathrm{h}^{-1} \mathrm{kpc}$. We point out that even if they do not constitute merger events, galaxies in the distant pair subsample might show disturbed morphologies (Ellison et al. 2008, 2013).

There are an additional 582 galaxies that do not fulfil any of the criteria defined above. They have not experienced any merger in the last 2 Gyr nor do they have any companion closer than $100 h^{-1} \mathrm{kpc}$. This subsample constitute a useful control sample of unperturbed galaxies.

Figure 1 shows examples of g-band mock images for major merger remnants, major close pairs and minor close pairs at $z=0$.

\section{MOCK IMAGES ANALYSIS}

\subsection{Image degradation}

Similarly to the procedure described by Snyder et al. (2015b), we transform the noise-free mock images in the gband to mimic the Sloan Digital Sky Survey (SDSS) main galaxy sample. Firstly, we assume that all images are at $z \sim 0.05$. Secondly, we convolve each idealized image with a Gaussian PSF with a full-width at half maximum (FWHM) of 1 arcsec simulating the effects of seeing. Thirdly, we re-bin the images to a constant pixel scale of 0.24 arcsec. Finally, we add Gaussian noise to the images such that the average signal-to-noise ratio of each galaxy pixel is 25 . Therefore, we simulate only strongly detected galaxies with morphological measurements not affected by noise. Figure 2 shows examples of mock images before and after the degradation procedure.

\subsection{Morphology measurements}

Each image is run through SEXTRACTOR in order to produce initial segmentation maps. We find that parameter values of DETECT_MINAREA $=50$, DETECT_THRESH $=0.6$, DEBLEND_NTHRESH $=32$ and DEBLEND_MINCONT $=0.9$ produce acceptable segmentation maps that are able to correctly isolate the central subhalo from other halo structures present in the mock images. No attempt is made to manually correct segmentations maps due to the large sample size. The third column of Fig. 2 shows examples of segmentation maps obtained using this procedure.

We evaluate each image background by selecting a 20 x 20 pixel square area where no structure was detected by the corresponding segmentation map. The background is removed from the image by subtracting the average pixel value of the background region from all pixels. Then we assign to the central subhalo all pixels belonging to the segmentation region that includes the central pixel of the image. Finally, the remaining pixels belonging to any other segmentation map are set to zero so no light from other structures besides the central subhalo affects the morphological measurements. Below, we briefly describe each of the morphological parameters used in this study.

The Petrosian radius $r_{p}$ is defined as the radius at which the ratio between the surface brightness and the mean surface brightness is equal to 0.2 . For each subhalo, we compute $r_{p}$ by adopting circular $\left(r_{p}^{c}\right)$ and elliptical $\left(r_{p}^{e}\right)$ apertures.

The asymmetry parameter $(A)$ is defined as a measure of the fraction of the light in non-symmetric components (Abraham et al. 1996; Conselice et al. 2000)

$A=\sum_{i, j} \frac{\left|I(i, j)-I_{180}(i, j)\right|}{|I(i . j)|}-B_{180}$,

where $I$ is the original image and $I_{180}$ is the image rotated by 180 degrees about a central pixel. The sum in equation 1 is done over all pixels within $1.5 r_{p}^{c}$ and the central pixel is determined by minimizing $A . B_{180}$ represents the average asymmetry of the background and is computed in the sky region defined above.

Typical $A$ values depend on morphology type, with ellipticals having $A \sim 0.02 \pm 0.02$ and spirals, in the range $A \sim 0.07-0.2$. Starburst galaxies such as Ultra- 


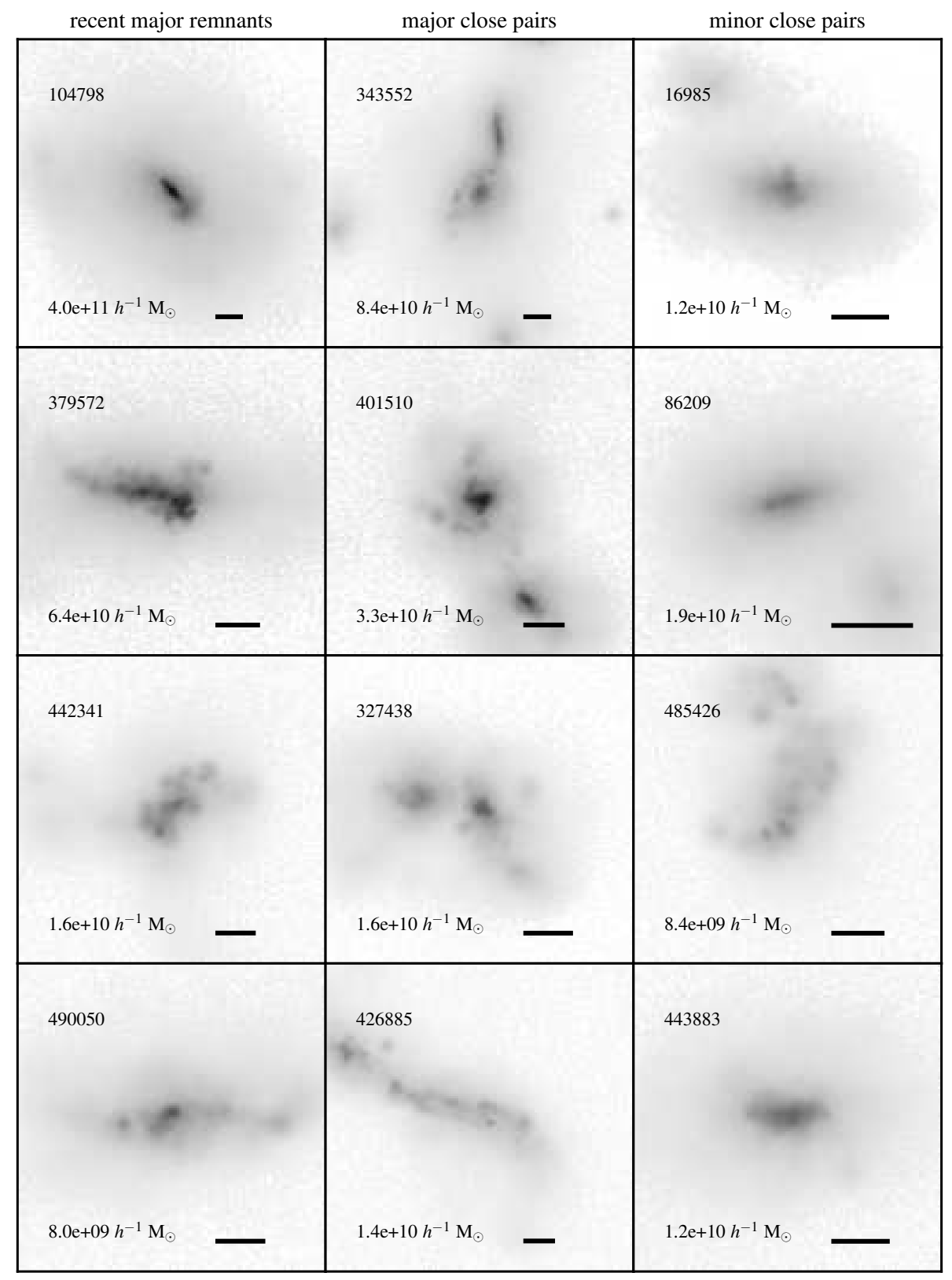

Figure 1. Examples of g-band mock images for major merger remnants (left column), major close pairs (central column) and minor close pairs (right column). The scale of the image is indicated by a $10 h^{-1} \mathrm{kpc}$ horizontal bar. Also shown are the subhalo identification number (id) and the stellar mass of the subhalo that appear at the centre of each image.

Luminous Infrared Galaxies (ULIRGs), which are often associated to major mergers, present values of $A \sim 0.32 \pm 0.19$ (Conselice et al. 2003).

The concentration $(C)$ measures the amount of light within the galaxy central region. It is defined as the ratio between the circular radii containing 20 percent and that corresponding to 80 percent of the total galaxy flux (Bershady et al. 2000):

$C=5 \log \left(\frac{r_{80}}{r_{20}}\right)$

Following standard procedures (Conselice et al. 2003;
Lotz et al. 2011), we compute the total flux within $1.5 r_{p}^{c}$ of the galaxy centre defined by the pixel that minimizes $A$.

The clumpiness $(S)$ quantifies the degree of small-scale structure (Conselice et al. 2003) and is defined as

$S=\sum_{i, j} \frac{\left|I(i, j)-I_{S}(i, j)\right|}{|I(i, j)|}-B_{S}$,

where $I_{S}$ is the image smoothed by a $2 \mathrm{D}$ boxcar of width 0.25 $r_{p}^{c}$ and $B_{S}$ is the average clumpiness of the background. Like $A$ and $C, S$ is also summed over $1.5 r_{p}^{c}$ but the central 0.25 $r_{p}^{c}$ region is excluded to avoid the extremely bright galactic cores.

The gini coefficient $(G)$ measures the degree of inequal- 


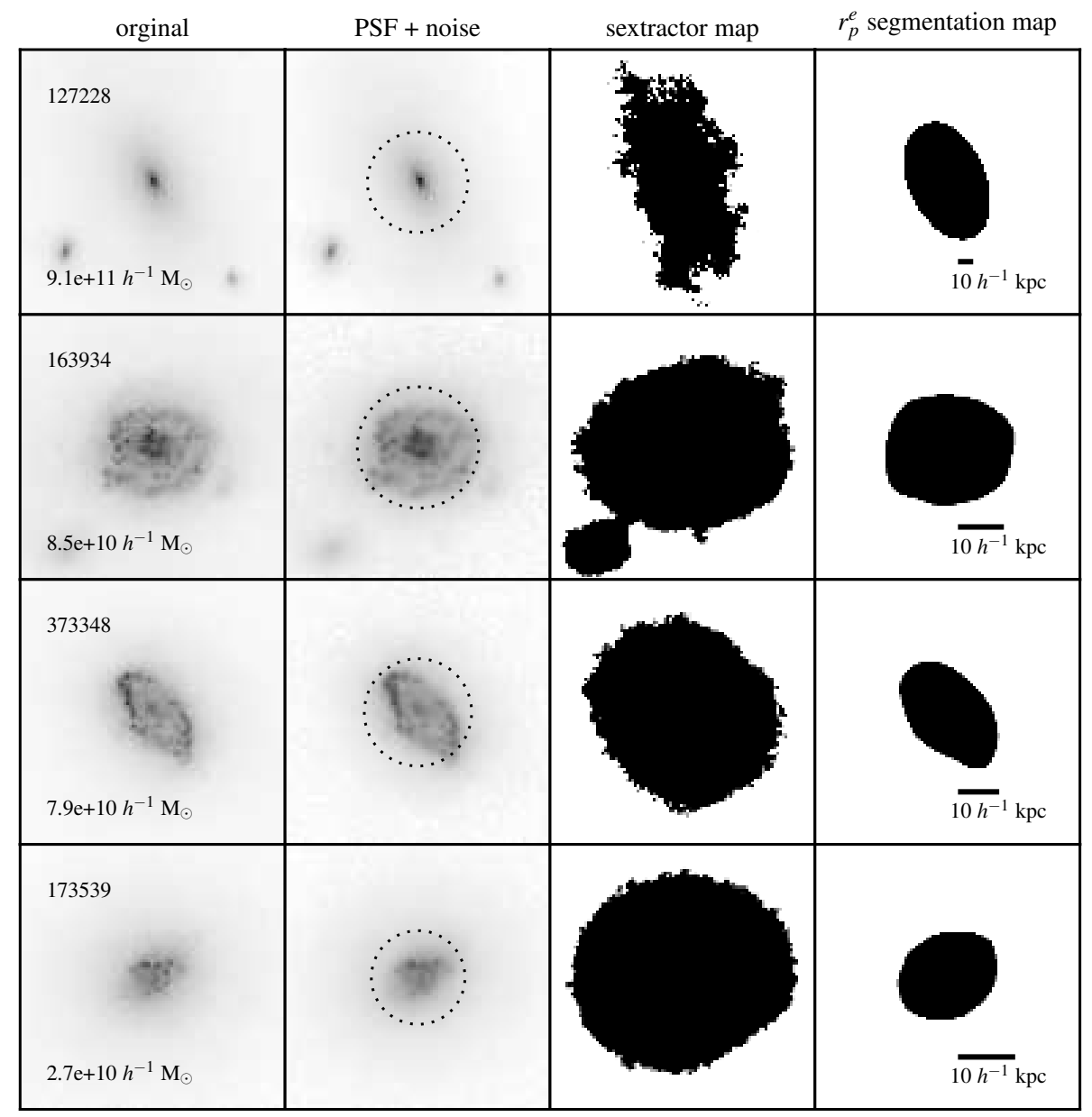

Figure 2. Example of g-band mock images before and after the image degradation procedure and resulting segmentation maps at $z=0$, arranged by increasing stellar mass from bottom to top. The first column shows the original images. The second column shows the resulting images after degradation, the dashed region represents the circular Petrosian radius. The third column shows the segmentation maps obtained from SEXTRACTOR and the final column displays the segmentation maps used to compute the $G$ and $M_{20}$ statistics as described in section 3.2 .

ity in the light distribution and is computed as

$G=\frac{1}{|\vec{X}| n(n-1)} \sum_{i}^{n}(2 i-n-1)\left|X_{i}\right|$,

where $X_{i}$ represent pixel values assigned to a galaxy, sorted into increasing flux order.

A $G$ coefficient of zero means that the galaxy light is evenly distributed among all pixels, while values approaching one imply that a few pixels concentrate most of the light. Unlike $C, G$ does not make any assumption regarding the underlying morphology and is therefore sensitive to regions of flux concentration outside the galactic centre.

Because the gini coefficient is very sensitive to which pixels are assigned to the galaxy, we follow a prescription similar to Lotz et al. (2004) to obtain additional segmentation maps that result in robust values of $G$. Starting from the central image pixel, we compute a binary segmentation mask employing an 8-connected structure detection algorithm. The mask is built by accepting all pixels that are 8 -connected to previously accepted pixels and have values above a given threshold. We use as threshold the average pixel value at a $r_{p}^{e}$ distance from the centre. The use of the elliptical Petrosian radius is fundamental to obtain consistent results at all galaxy orientations. The last column of Fig. 2 shows examples of segmentation maps obtained from the described procedure (see appendix A for a comparison with Lotz's results).

The total second-order moment $M_{\text {tot }}$ is defined as

$M_{\mathrm{tot}}=\sum_{i}^{n} M_{i}=\sum_{i}^{n} I_{i}\left(\left(x_{i}-x_{c}\right)^{2}+\left(y_{i}-y_{c}\right)^{2}\right)$

where $I_{i}$ is the flux in each pixel, $\left(x_{i}, y_{i}\right)$ represent individual pixel coordinates and $\left(x_{c}, y_{c}\right)$ denotes the galaxy centre. The sum is performed over all pixels assigned to the galaxy by the same segmentation map used to compute the $G$ index. The centre is computed by finding $\left(x_{c}, y_{c}\right)$ such that $M_{\text {tot }}$ is minimized.

The second order moment of the brightest pixels of a galaxy is sensitive to the spatial distribution of bright nuclei, spiral arms, bars and off-centre star clusters. $M_{20}$ is defined as the normalized second order moment of the brightest 20 per cent of a galaxy flux. To compute $M_{20}$, we sort the galaxy pixels in descending order of flux, sum $M_{i}$ until the value equals 20 percent of the total galaxy flux and then normalize 


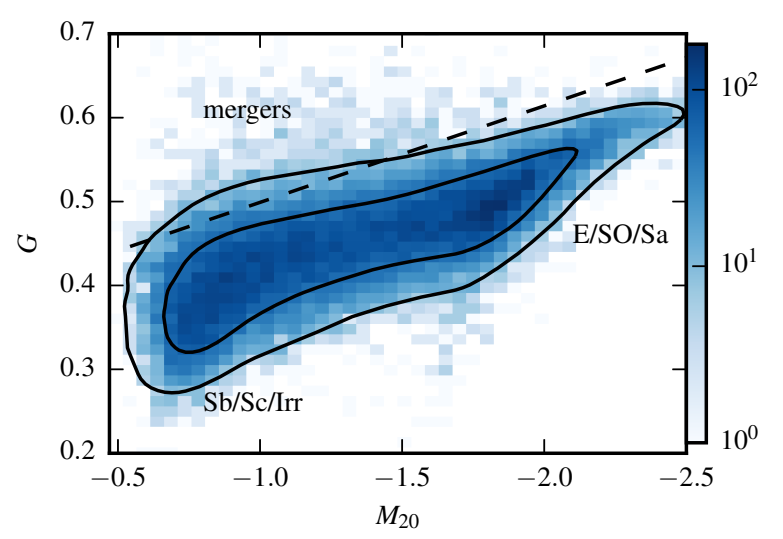

Figure 3. $G-M_{20}$ relation for the full I-1 galaxy sample. The colorbar indicates the number of galaxies in each bin. Perturbed morphologies are located above the LPM04 empirical demarcation line (shown as a dashed line). Bulge-dominated galaxies are found towards the upper right corner while disc-dominated galaxies are located in the lower left corner. Solid lines mark the regions which enclose 95 and 68 percent of subhalos.

by $M_{\text {tot }}$ :

$M_{20}=\log _{10}\left(\frac{\sum_{i} M_{i}}{M_{\text {tot }}}\right)$, while $\sum_{i} f_{i}<0.2 f_{\text {tot }}$.

The normalization by $M_{\text {tot }}$ removes the dependence on total galaxy flux.

According to equation $6, M_{20}$ is always a negative quantity. For normal early-type galaxies typical $M_{20}$ values are $\sim-2$, while for late-type galaxies, $M_{20} \sim-1.5$. It has been shown that mergers present higher values, $M_{20} \geq-1$, specially those with multiple nuclei (Lotz et al. 2008a).

\section{ANALYSIS}

\section{1 $G-M_{20}$ criteria}

In Fig. 3 we show the $G-M_{20}$ statistics distribution for our complete I-1 galaxy sample. Our computations reproduce the morphological trends found by Snyder et al. (2015b): quenched, bulge-dominated galaxies have large $G(\sim 0.6)$ and low $M_{20}(\sim-2.5)$ values, while disc-dominated galaxies have lower $G(<0.5)$ and higher $M_{20}(>-1)$ values. We point out that the mock images employed in the present work differ from the ones used in Snyder et al. (2015b) in that they include all material from the FOF halo that falls within the mock image FOV (see Section 2.3 for details). For individual galaxies, we find that our $G-M_{20}$ statistics generally differ less than 10 percent from the one reported by Snyder et al. (2015b).

In Fig. 4, we analyse the G-M $\mathrm{M}_{20}$ morphologies for galaxies in the merger remnants and pairs subsamples selected from the I-1 simulation. We find that the morphological indicators for major merger remnants and close pairs differ the most from those found in the full sample, as expected for ongoing mergers, since they present disturbed morphologies. LPM04 found that visually classified mergers in the
Table 1. Merginess frequency. Number and percentages of mock galaxies above and below the LPM04 empirical merger demarcation line for merger remnants, galaxy pairs and unperturbed galaxies. Each class has been normalized to the total number of members in the subsample. All four cameras are included.

\begin{tabular}{lrrrr}
\hline Class & \multicolumn{2}{c}{ Merginess $\geq 0$} & \multicolumn{2}{c}{ Merginess $<0$} \\
& $\mathrm{~N}$ & Percentage & $\mathrm{N}$ & Percentage \\
\hline Close pairs & 300 & 10.1 & 2676 & 89.1 \\
Major mergers & 90 & 7.0 & 1198 & 93.0 \\
Minor mergers & 635 & 4.2 & 14437 & 95.8 \\
Distant pairs & 607 & 3.0 & 19577 & 97.0 \\
Unperturbed & 38 & 1.6 & 2290 & 98.4 \\
galaxies & & & & \\
\hline
\end{tabular}

Borne et al. (2000) observations of local ULIGRs could be separated from normal galaxies by

$G>-0.115 M_{20}+0.384$.

This relation is displayed in Fig. 3 as well as in all panels of Fig. 4 and constitutes an empirically derived demarcation line to separate irregular and disturbed morphologies, often caused by mergers, from normal unperturbed galaxies.

Following Snyder et al. (2015b), we define the merginess as the perpendicular distance to the LPM04 demarcation line. We assign positive (negative) values to points above (below) this line. The merginess provides a qualitative estimation of the level of morphological disturbance present. As can be seen in Fig. 4, and in Table 1 a significant number of close pairs have positive merginess, indicating the presence of disturbed morphologies, while all other samples present a lower proportion of galaxies in the merger zone. From Table 1 a clear hierarchy in the proportion of galaxies with positive merginess can be found, with close pairs presenting the highest percentage $(10.1 \%)$, follow by major mergers remnants $(7.0 \%)$, minor merger remnants $(4.2 \%)$, and distant pairs $(3.0 \%)$. Lastly, the unperturbed isolated galaxies sample have the lowest proportion of galaxies above the demarcation line (1.6\%).

Close pairs appear clustered at $\mathrm{M}_{20}>-1$ consistent with the detection of multiple nuclei within the segmentation map and comparable to the values found by Lotz et al. (2004) for double and multiple nuclei ULIRGs, which present higher $\left(\mathrm{M}_{20} \sim-1\right)$ values that single nuclei ULIRGs $\left(\mathrm{M}_{20} \sim-2\right)$.

\subsection{Merger remnants}

According to Lotz et al. (2010a), $G-M_{20}$ morphologies are particularly sensitive to mergers with baryonic mass ratios between 1:1 and 10:1, during time-scales lasting 0.2-0.4 Gyr. The disturbed morphologies are more noticeable during the close approaches and the final merger stages. They also find that major merger remnants observed after more than 1 Gyr of the event present morphologies similar to early-type spirals while minor mergers are found to have minimal effects on the $G$ and $M_{20}$ values of their remnants.

In Fig. 5 we show the mean merginess of galaxies as a function of time since the last merger event. We find that the morphological disturbance is larger for galaxies having just experienced a major merger (blue triangles). A very good correlation signal is found between the merginess and the 


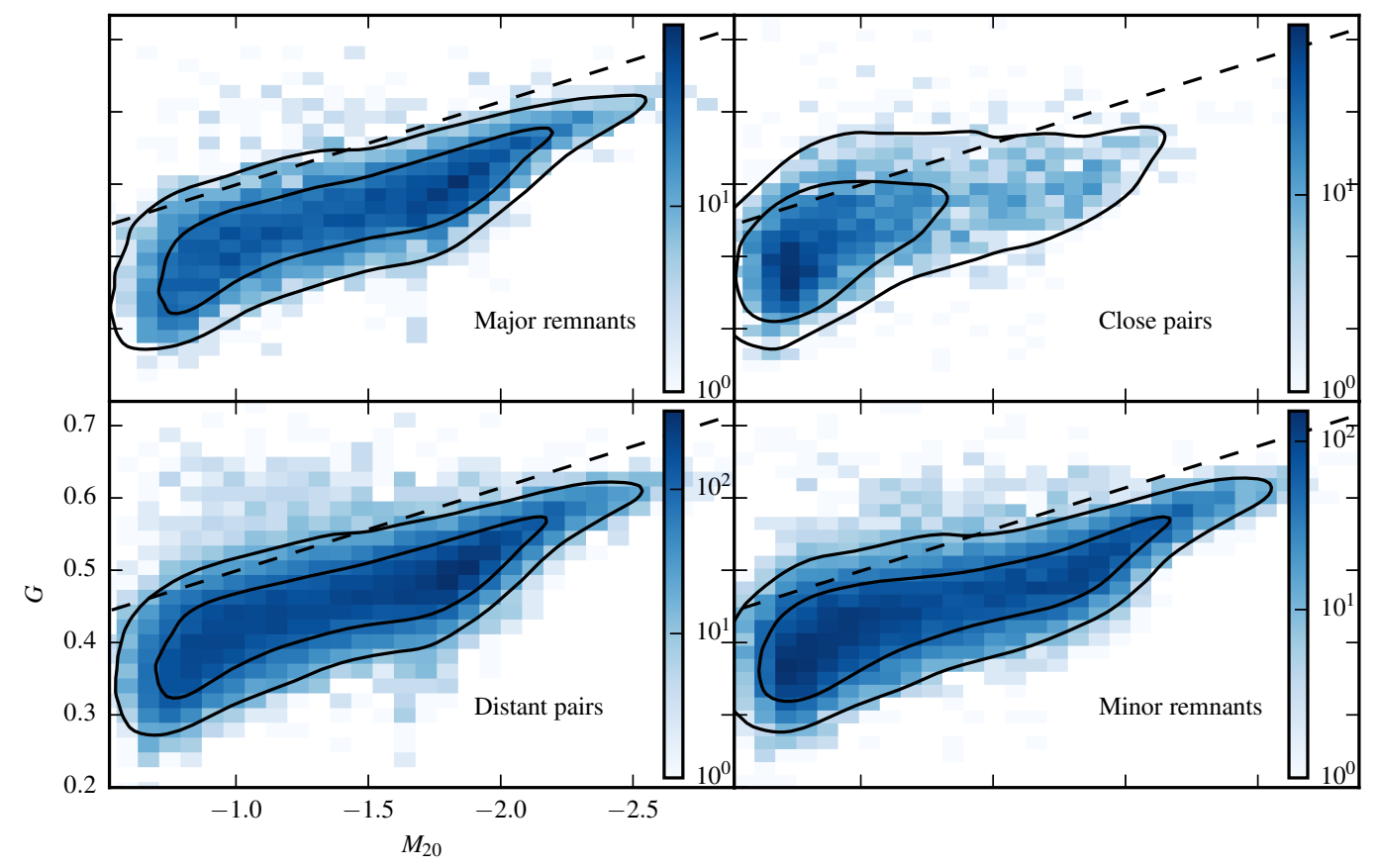

Figure 4. $G-M_{20}$ relation for galaxies that experienced a major merger in the last 2 Gyr (upper left panel), galaxies having a close companion at a distance $\mathrm{d}<20 h^{-1} \mathrm{kpc}$ (upper right panel), galaxies having a close companion at a distance $20 h^{-1} \mathrm{kpc}<\mathrm{d}<100$ $h^{-1} \mathrm{kpc}$ (lower left panel) and galaxies that experienced a minor merger in the last 2 Gyr (lower right panel). Colorbars indicate the number of galaxies in each bin. The dashed line separates mergers from normal galaxies according to LPM04. Solid lines mark regions which enclose 95 and 98 percent of subhalos.

elapsing time since the last major merger. Events occurring more than 2 Gyr ago show mean merginess comparable to the average value of the unperturbed sample of galaxies. Spite of the fact that the mean merginess remains negative for all time, the good correlation signal shows that it is still possible to statistically classify recent mergers by using the $G-M_{20}$ statistic.

The mean merginess for minor mergers $\left(0.1<\mu_{*} \leq\right.$ $0.25)$ is also shown in Fig. 5 as green circles. This subsample of galaxies is noisier and has lower values for a given elapsing time to that of major mergers, but shows a similar correlation with time, indicating that the $G-M_{20}$ is still sensitive to mergers in this mass ratio range. In contrast, the mean merginess for very minor mergers $\left(0.01<\mu_{*} \leq 0.1\right)$ shows a much weaker dependence with time, corroborating the result found by Lotz et al. (2008a) which suggested that very minor mergers do no significantly affect the final values of $G$ an $M_{20}$ of their remnants. The dashed lines in Fig. 5 represent linear regression fits to the corresponding mean merginess. The parameters of the fitting are summarized in Table 2

To visualize how the merger remnants are located in $G-M_{20}$ plane according to the elapsing time to the last merger event, we displayed them in Fig. 6. As expected from our previous discussion, recent major mergers are more likely to be found above the empirical demarcation line than older merger remnants. This is also in agreement with Lotz et al. (2010a). findings which indicate that more recent merger remnants are more likely to be classified positively. However, we note that a large number of major merger remnants are located below the demarcation line even for very recent

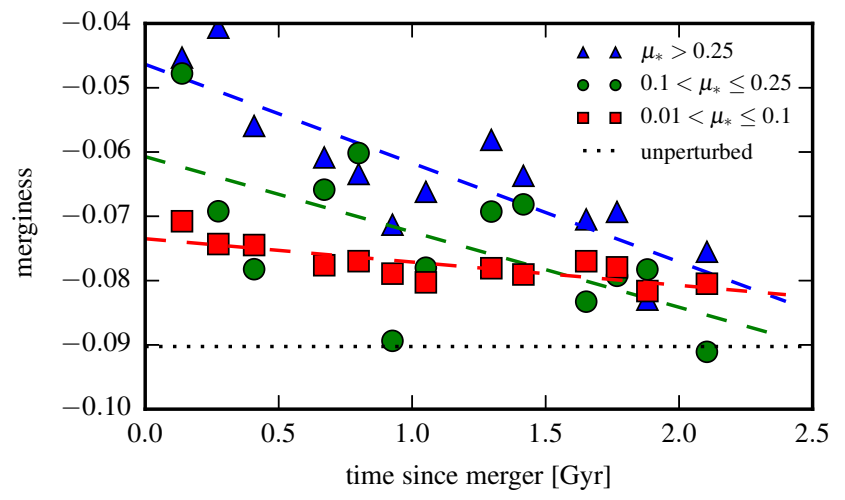

Figure 5. Mean merginess as a function of elapsing time since the last major merger $\left(\mu_{*}>0.25\right.$; blue triangles $)$, minor mergers $\left(0.1<\mu_{*} \leq 0.25\right.$; green circles $)$ and very minor mergers $\left(0.01<\mu_{*} \leq 0.1\right.$; red squares $)$. The dashed lines represent linear regressions fitting to the corresponding data. For comparison we included the mean merginess of the unperturbed sample (horizontal dotted line).

merger. This is consistent with previous findings suggesting that the $G-M_{20}$ method yields an incomplete classification (Kampczyk et al. 2007).

Interestingly, we also find that there is a shift in the position of merger remnants with time along the relation, such that very recent mergers appear to be clustered around $M_{20} \sim-1.5$ while older remnants are shifted towards lower 
Table 2. Parameters of the linear regression fits of the mean merginess as a function of the elapsing time to the last merger event for galaxy mergers with different mass ratios $\mu_{*}$ (Fig. 5).

\begin{tabular}{lcc}
\hline Mass ratio $\mu_{*}$ & $\mathrm{a}$ & $\mathrm{b}$ \\
& $\operatorname{dex} \mathrm{Gyr}^{-1}$ & $\mathrm{dex}$ \\
\hline$\mu_{*} \geq 0.25$ & $-0.015 \pm 0.003$ & -0.04 \\
$0.25<\mu_{*} \leq 0.1$ & $-0.012 \pm 0.003$ & -0.006 \\
$0.1<\mu_{*} \leq 0.01$ & $-0.004 \pm 0.001$ & -0.07 \\
\hline
\end{tabular}

values $\left(M_{20} \sim-2\right)$. As mentioned before, higher $M_{20}$ values are an indication of multiple nuclei detected within a segmentation map and are expected to appear in the time immediately after the final encounter. At intermediate times $(\mathrm{t} \sim 1$ Gyr) a tail towards bulge dominated galaxies can be found. While, at late stages $(\mathrm{t} \sim 2 \mathrm{Gyr})$ major remnants are found in the zone between late type galaxies and bulge dominated early type galaxies indicating that despite the major merger, many galaxies manage to retain or recover their disc structures. This result agrees well with previous findings by Robertson et al. (2006) that gas-rich mergers can form rotationally supported gaseous structures from residual angular momentum after the final coalescence, with similar trends found in zoom-in merger simulation by Snyder et al. (2015a) and with results found by Lotz et al. (2008a) where equal mass, gas rich isolated merger simulations appear disc-like when observed $t>1$ Gyr after the final coalescence.

We next determine the effectiveness of the LPM04 empirical demarcation criteria to identify major merger remnants.

For a given selection criteria such as the LPM04 empirical demarcation line, it is necessary to ascertain how effectively it selects a given subsample of galaxies. In a similar way to the method used by Huertas-Company et al. (2014) to calibrate automatic proxies of galaxy morphology we proceed to define the following quantities:

(i) True positives (TP): Number of galaxies selected by the criteria which belong to the subsample.

(ii) True negatives (TN): Number of galaxies not selected by the criteria which do not belong to the subsample.

(iii) False positives (FP): Number of galaxies selected by the criteria which do not belong to the subsample.

(iv) False negatives (FN): Number of galaxies not selected by the criteria which belong to the subsample.

We define two additional quantities: the purity $(\mathrm{P})$ and the completeness (Cp). Purity is the percentage of selected galaxies that belong to the subsample among all galaxies selected by the criteria.

$\mathrm{P}=100 \times \frac{T P}{F P+T P}$.

It effectively measures the level of contamination. For example, if 90 per cent of galaxies with positive merginess have in fact not experienced a major merger, then the purity of the LPM04 criteria in selecting major merger remnants will be 10 per cent.

The completeness $(\mathrm{Cp})$ is the percentage of selected galaxies which belong to the subsample among all galaxies belonging to the subsample.

$\mathrm{Cp}=100 \times \frac{T P}{F N+T P}$.

For example, if all major merger remnants have positive merginess, then the completeness of the LPM04 criteria in selecting major merger remnants will be 100 per cent.

We performed a visual inspection of the galaxies presenting positive merginess in the first of the four cameras in order to determine the possible presence of sources of contamination that would affect the computations of $\mathrm{P}$ and Cp. From the original 266 galaxies we find that 4 (1.5\%) galaxies are edge-on, a common source of confusion for the G- $\mathrm{M}_{20}$ criteria, $43(16.2 \%)$ present ring-like structures of recent star formation previously reported by Snyder et al. (2015b); Torrey et al. (2015), finally we found 50 (18.8\%) galaxies with irregular and starbursting appearance, but no sign of recent or current interaction such as tidal tails or a close companion. These galaxies have low stellar mass $\left(M_{*} \sim 10^{10} \mathrm{M}_{\odot}\right)$ and are likely the result of stochastic recent star formation which combined to the limited numerical resolution produced artificially large star formation regions. We also find similar trends in the rest of the cameras. We define a clean sample of perturbed galaxies as the galaxies that present positive merginess in the first camera once we remove the above sources of contamination.

In Fig. 7 we show the dependence of the $\mathrm{Cp}$ and $\mathrm{P}$ quantities on the elapsing time since the last merger event using the clean sample defined above. From the completeness we find that about 12 per cent of major mergers occurring since $t<0.14$ Gyr are classified as perturbed according to the LPM04 empirical criterium. This percentage decreases towards 5 per cent at $t \sim 1$ Gyr where it remains approximately constant afterwards. This result further corroborates that this empirical criteria is sensitive to major mergers occurring less than 1 Gyr ago and specially sensitive to very recent events. The purity indicates a high level of contamination for all elapsing times, which is expected because at a given time, only a small fraction of perturbed galaxies are expected to be a major merger remnant. We can conclude that $\sim 5$ per cent of galaxies with positive merginess can be explained as a major merger remnant. Figure 7 also shows $\mathrm{Cp}$ and $\mathrm{P}$ for merger remnants with $\mu_{*}>0.1$, completeness values present a similar dependence with the elapsing time since the merger event compared to major mergers. After 1 Gyr completeness levels off showing that the $G-M_{20}$ criteria is still sensitive to $\mu_{*}>0.1$ younger than $1 \mathrm{Gyr}$.

\subsection{On-going mergers}

While our merger sample selected by using the merger trees represents the remnants of mergers, our pair sample represents the population of on-going interactions. In Fig. 8 we show the distribution of relative distances between pairs for those galaxies with positive and negative merginess. There is a clear excess of close pairs with perturbed morphologies. As it has been pointed out in previous works, the largest morphological changes and star formation excess are, detected for galaxies within $\sim 35 \mathrm{~h}^{-1} \mathrm{kpc}$ in both observational (e.g. Barton et al. 2000; Lambas et al. 2003; Ellison et al. 2008; Scudder et al. 2012) and numerical (e.g. Perez et al. 2006; Di Matteo et al. 2007) studies. 


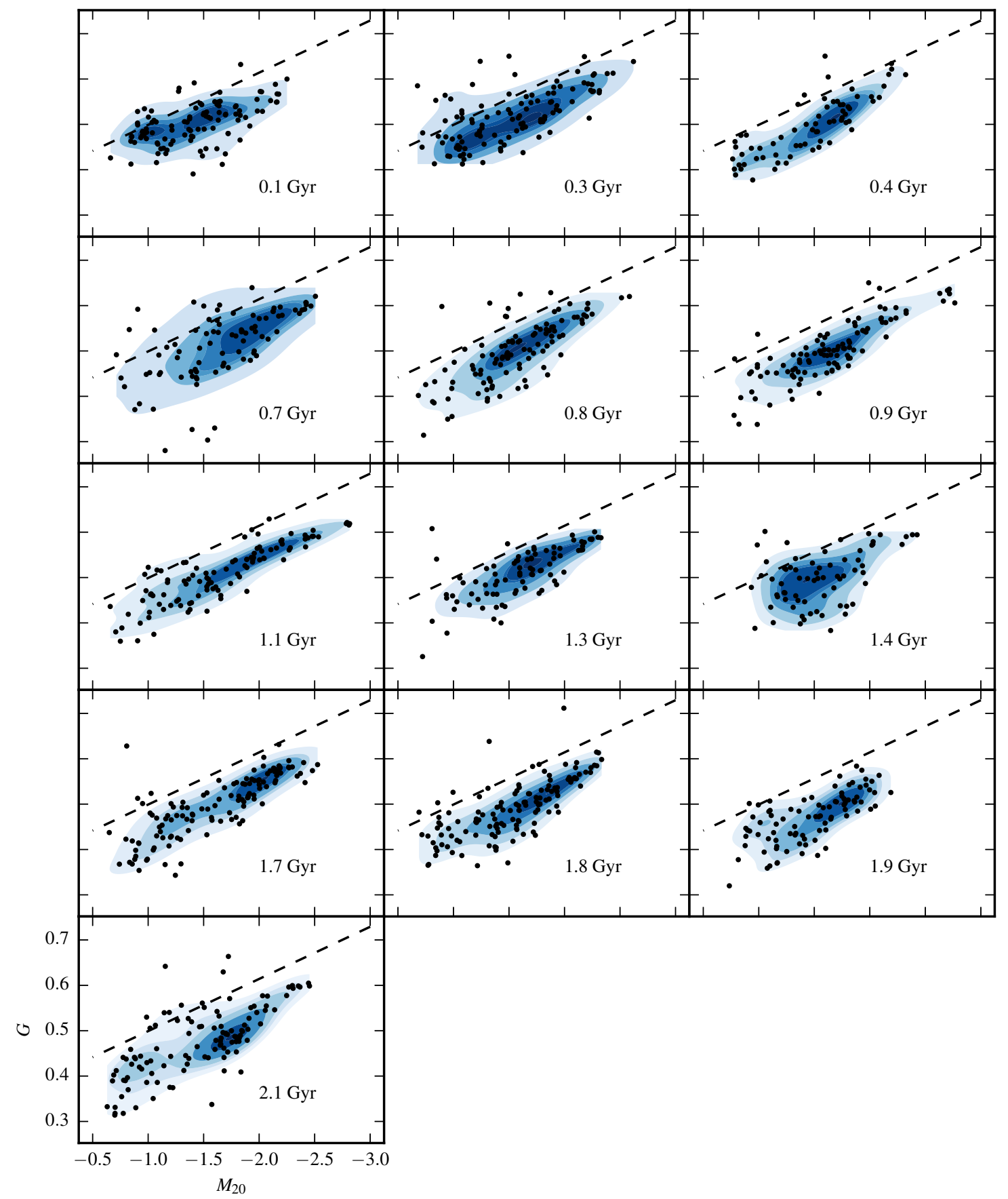

Figure 6. $G-M_{20}$ morphologies for galaxies having experienced the last major merger event at increasing times for each panel. Points represent the major merger remnants, while the shaded contours denote regions that enclose 90, 70, 50, 30 and 20 per cent of remnants (from light blue to dark blue colours). The dashed line corresponds to the LMP04 demarcation line.

In Fig. 9 we show the purity and completeness of the cleaned sample as a function of distance between pair members. When considering all mass ratios $\left(\mu_{*}>0.001\right)$, we find low completeness values $(\sim 5 \%)$ at all separations. Conversely, for $\mu_{*}>0.1$ we find completeness values that reach $\sim 40 \%$ at $d<20 h^{-1} \mathrm{kpc}$. This confirms that the $G-M_{20}$ criterium is sensitive to mass ratios larger than 0.1 . We notice that completeness increases by a factor of 1.3 for major mergers with $d<20 h^{-1} \mathrm{kpc}$, supporting the claim that
$G-M_{20}$ is more sensitive to this kind of events. Apart from this, the behaviour is very similar to that determined by imposing a limit at $\mu_{*}=0.1$ as reported by Lotz et al. (2010a).

Purity for pairs with $\mu_{*}>0.1$ plateaus at $\sim 50 \%$ for separations greater than $\sim 100 h^{-1} \mathrm{kpc}$. We adopt this percentage as the fiducial value for contamination of our clean sample. This value is of fundamental importance for our derivation of the merger rate. 


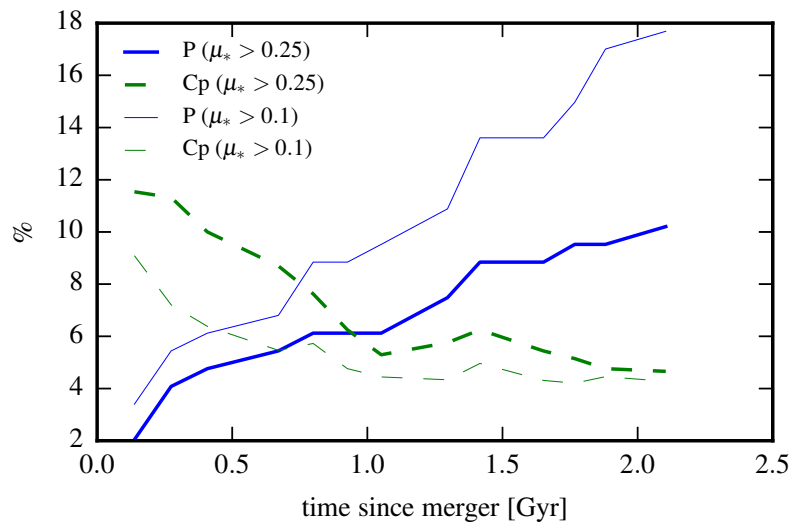

Figure 7. Purity (solid line) and completeness (dashed line) for merger remnants as a function of elapsing time since the last merger event. Thick lines denote remnants with $\mu_{*}>0.25$, while thinner lines denote remnants with $\mu_{*}>0.1$.

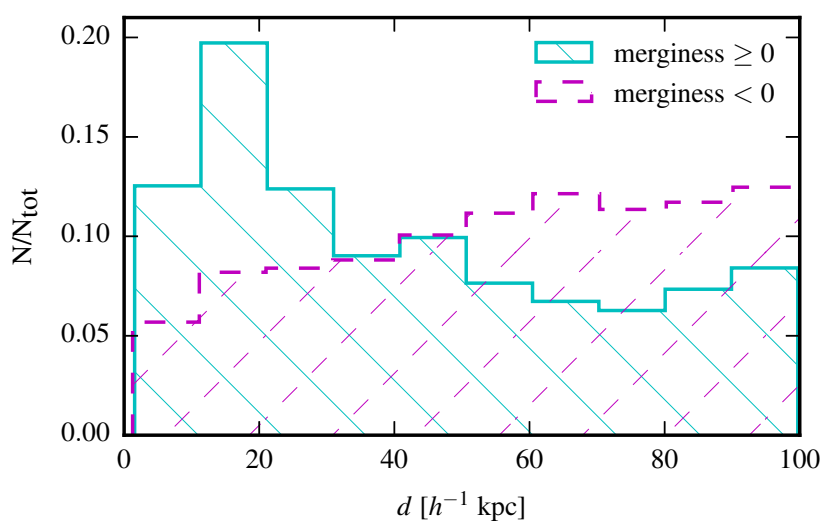

Figure 8. Distribution of relative distances between galaxies in pairs with positive merginess (cyan solid lines) and negative merginess (magenta dashed line).

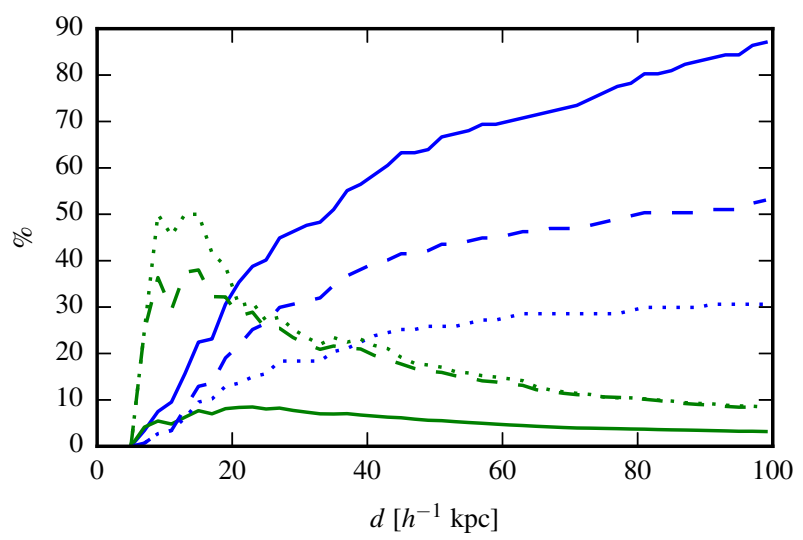

Figure 9. Purity (blue lines) and completeness (green lines) for pairs as a function of relative distance between galaxy members when considering the following mass ratios: $\mu_{*}>0.25$ (dotted lines), $\mu_{*}>0.1$ (dashed lines), $\mu_{*}>0.001$ (solid lines)
Table 3. Number and percentages of galaxies classified according to the criteria proposed by Conselice (2003).

\begin{tabular}{lllll}
\hline Class & \multicolumn{2}{c}{$A \geq 0.35$} & \multicolumn{2}{c}{$A<0.35$} \\
& $\mathrm{~N}$ & Percentage & $\mathrm{N}$ & \begin{tabular}{l} 
Percentage \\
\hline Major mergers
\end{tabular} \\
580 & 45 & 708 & 55 \\
Close pairs & 2356 & 79.2 & 620 & 20.8 \\
Minor mergers & 7721 & 51.2 & 7351 & 48.8 \\
Pairs & 8468 & 42 & 11716 & 58 \\
Unperturbed & 955 & 41 & 1373 & 59 \\
galaxies & & & & \\
\hline
\end{tabular}

\subsection{Asymmetry criteria}

The Asymmetry parameter is also commonly used to classify merger candidates. The calibration for local mergers by Conselice (2003) finds the following merger criterium

$A \geq 0.35$.

In Fig. 10 we show concentration vs. asymmetry for galaxies in the I- 1 simulation at $z=0$. As before we treat each of the four cameras an independent measurement. We discriminate again between major mergers, minor mergers close pairs, and distant pairs. We find that the condition $A \geq 0.35$ roughly divides in half the galaxy populations considered with the exception of close pairs which present a significant excess of asymmetry. From this figure, then, it is clear that close pairs are those better classified by the asymmetry criterium.

As can be seen in Fig. 11, the mean asymmetry of galaxies increases with decreasing mass, resulting in a large fraction of subhalos with stellar masses $10^{10} \mathrm{M}_{\odot}$ having $A \geq 0.35$. As mentioned before, galaxies with masses around $10^{10} \mathrm{M}_{\odot}$ show a trend towards having more irregular morphologies due to stochastic star formation and limitations of the numerical resolution of the simulation. Hence, in order to better assess the behaviour of $A$ for galaxy pairs, the subsample is divided in mass intervals.

Regardless of stellar mass, the mean asymmetry of subhalos increases for close pairs, as can be seen in Fig. 12 where we show $\langle A\rangle$ as a function of distance to the closest companion galaxy for mass bins in the ranges $11<\log _{10} M_{*} \leq 11.5$, $10.5<\log _{10} M_{*} \leq 11$ and $10<\log _{10} M_{*} \leq 10.5$. We also include the relation for the whole sample $\left(10<\log _{10} M_{*} \leq\right.$ 11.5). All mass bins show an increase of the $\langle A\rangle$ over the reference value at $d \sim 30 h^{-1} \mathrm{kpc}$

The complete sample shows an increase of the $\langle A\rangle$ over the reference value at $d \sim 30 h^{-1} \mathrm{kpc}$. Galaxies with $\log _{10} M_{*}>11.0$ show the sharper increases but for galaxies at lower separation, $d \sim 20 h^{-1} \mathrm{kpc}$ and for larger pair separations $\langle A\rangle$ is lower than 0.2. As we take smaller galaxies, the $\langle A\rangle$ are higher and show a weaker variation with the relative distance between pairs. However, smaller galaxies get to values larger than $\langle A\rangle \sim 0.35$ at larger pair separations. The smaller mass interval might be more affected by resolution problems which produce spurious signals of disturbances in the non-parametric morphologies. However, the trend is present progressively as one moves from higher mass to lower mass.

In order to explore further if the increase of asymme- 


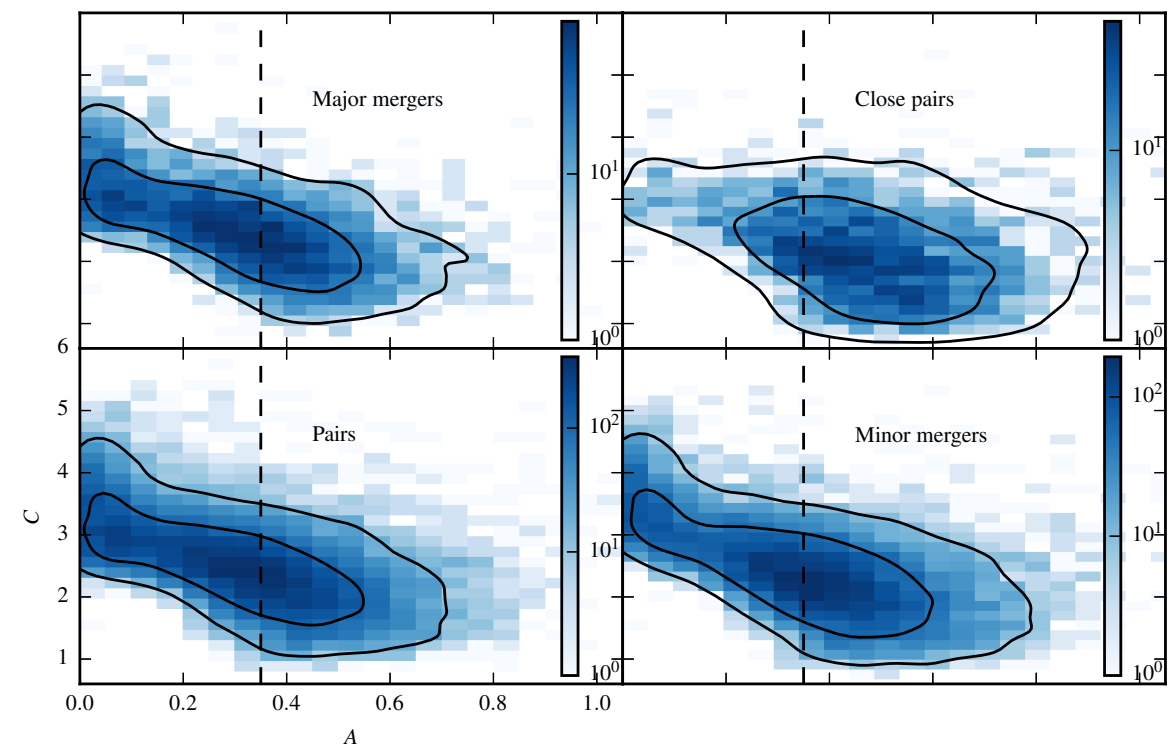

Figure 10. $A-C$ relation for galaxies that experienced a major merger in the last 2 Gyr (upper left panel), for galaxies having a close companion at a distance $d<20 h^{-1} \mathrm{kpc}$ (upper right panel), for galaxies having a close companion at a distance $d<100 h^{-1} \mathrm{kpc}$ (lower left panel) and for : galaxies that experienced a minor merger in the last 2 Gyr (lower right panel) in the I- 1 simulation at $z=0$. The vertical line separates mergers from normal galaxies according to empirical criteria of Conselice (2003). The contours mark regions that enclose 90, 70, 50, 30 and 20 percent of subhalos (from dark to light blue, respectively).

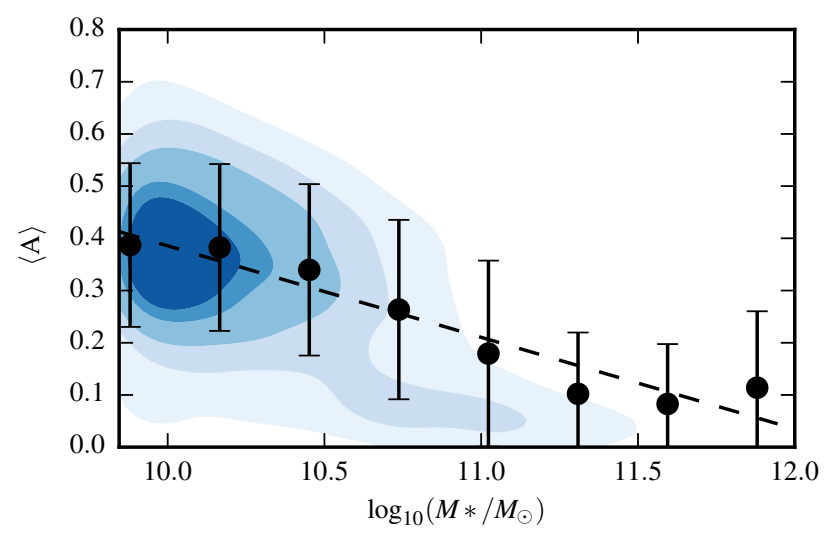

Figure 11. Mean asymmetry $A$ as a function of stellar mass. The contours mark regions that enclose 90, 70, 50, 30 and 20 percent of subhalos. The black dots show the mean asymmetry in stellar mass bins, with error bars representing the standard dispersion. The dashed line represents a linear fit to the points with slope $-0.17 \pm 0.02$ and intercept $2.14 \pm 0.01$.

try in close pairs is produced by physical disturbances in the galaxies such as tidal tails or induced star formation and not by light contamination from the secondary galaxies, we study the asymmetry as a function of relative velocity between pair members. This analysis will also allow us to detect the role play by those pairs with larger velocity separations which have a higher probability to be flyby events. As can be seen in Fig. 13, there is an increase in mean asymmetry over the reference value for close pairs having a relative velocity smaller that $\sim 400 \mathrm{~km} \mathrm{~s}^{-1}$ as is expected for interacting pairs (e.g Lambas et al. 2003)

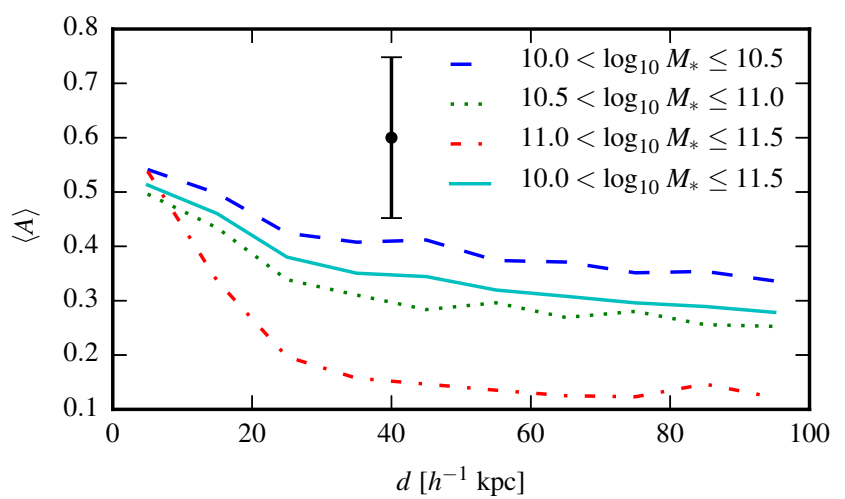

Figure 12. Mean asymmetry as a function of the relative distance to a companion galaxy for different stellar mass intervals: $10<\log _{10} M_{*} \leq 10.5$ (blue dashed line), $10.5<\log _{10} M_{*} \leq 11$ (green dotted line) and $11<\log _{10} M_{*} \leq 11.5$ (red dasheddotted line). The solid cyan line represents the full mass range $10<\log _{10} M_{*}<11.5$. The data point with error bars indicate the typical dispersion at a given pair separation.

For larger relative velocities, the asymmetry gets to value of around $\langle A\rangle \sim 0.1-0.2$. As galaxy pairs with larger separations are incorporated, the signal of anti-correlation decreases. However, the subsample of closer galaxy pairs clearly show the correlation between disturbances and mergers and suggests that flyby events will not be able to produce such a significant impact, on average.

Fig. 14 shows the dependence of purity and completeness on pair separation for the asymmetry merger criterium. 


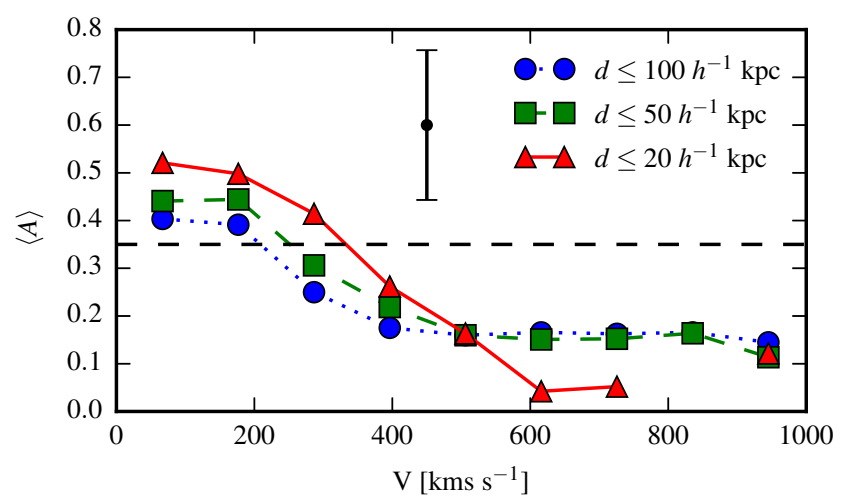

Figure 13. Mean asymmetry as a function of relative velocity between galaxy pairs for three pair separation. The horizontal dashed line shows the reference value $A=0.35$. The data point with error bars indicate the typical mean standard deviation. This trend shows the very weak effects of flybys in triggering a morphology perturbation.

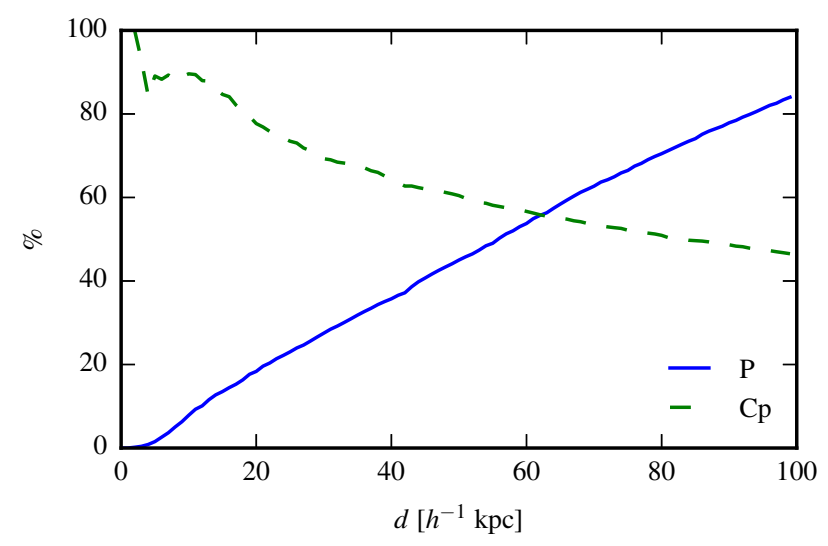

Figure 14. $A-C$ purity (solid line) and completeness (dashed line) for for galaxy pairs as a function of pair separation.

We find that the completeness increases from 60 per cent for $d<60 h^{-1} \mathrm{kpc}$ to 90 per cent within $d<10 h^{-1} \mathrm{kpc}$.

\section{GALAXY MERGER RATE}

Following Lotz et al. (2011) we define the volume-averaged galaxy merger rate $\Gamma_{\text {merg }}$ as the number of ongoing merger events per unit comoving volume $\left(\phi_{\text {merg }}\right)$, divided by the time $T_{\text {merg }}$ between the initial encounter and the final merger stage.

$\Gamma_{\mathrm{merg}}=\frac{\phi_{\mathrm{merg}}}{T_{\mathrm{merg}}}$

The number density of galaxies classified as galaxy mergers depends on the average time-scales $\left(\left\langle T_{\text {obs }}\right\rangle\right)$ during which the galaxy can be identified by some of the morphological methods discussed above, such that

$\phi_{\text {merg }}^{\prime}=\phi_{\text {merg }} \frac{\left\langle T_{\mathrm{obs}}\right\rangle}{T_{\mathrm{merg}}}$.

The galaxy merger rate can be calculated from the observed number density of galaxy merger candidates as

$\Gamma_{\text {merg }}=\frac{\phi_{\text {merg }}^{\prime}}{\left\langle T_{\text {obs }}\right\rangle}$.

Instead of $\phi_{\text {merg }}$, many authors estimate the fractional merger rate $R_{\text {merg }}$ defined as

$R_{\mathrm{merg}}=\frac{f_{\mathrm{merg}}}{\left\langle T_{\mathrm{obs}}\right\rangle}$

where $f_{\text {merg }}$ is the fraction of galaxies identified as mergers for a given galaxy sample. We can relate $\phi_{\text {merg }}^{\prime}$ to $f_{\text {merg }}$ by using:

$\phi_{\text {merg }}^{\prime}=f_{\text {merg }} n_{\text {gal }}$,

where $n_{\text {gal }}$ is comoving number density of galaxies.

A correction factor can be applied to the merger fraction to account for contamination from objects that are not mergers, such that

$f_{\text {merg }}=C_{\text {merg }} f_{\text {merg }}^{\text {obs }}$,

where $f_{\text {merg }}^{\text {obs }}$ is the fraction of galaxies identified as mergers before the correction is applied.

\subsection{Average merger observability time-scale}

Individual observability time-scales $T_{\text {obs }}$ were calculated by Lotz et al. (2008a, 2010b,a) for a suite of N-body/SPH isolated merger simulations spanning a range of galaxy masses, mass ratios, gas fractions, orientations and orbital parameters. SDSS-g mock images were used to calculate the time during which particular merger simulations would be counted as perturbed according to the $G-M_{20}$ criterium. They found that observability time-scales depend mostly on the mass ratio and gas fraction of galaxies involved in the merger, while orientation, orbital parameters and the final merger mass had little impact on $T_{\text {obs }}$.

Following Lotz et al. (2011) we compute the average observability time-scale $\left\langle T_{\text {obs }}\right\rangle$ expected for the I- 1 simulation at $z=0$ as:

$\left\langle T_{\mathrm{obs}}\right\rangle=\sum_{i, j} w_{i, j} \times T_{i, j}$

where $w_{i, j}$ is the fraction of mergers at $z=0$ with stellar mass ratio $i$ and baryonic gas fraction $j$, and $T_{i, j}$ is the observability time-scale corresponding to mergers with stellar mass ratio $i$ and baryonic gas fraction $j$.

Figure 15 shows the normalize distribution of stellar mass ratios and gas fractions for I- 1 mergers at $z=0$. We also estimated them for the same parameter space explored by the isolated merger simulations detailed above: $1-1 / 2$, $1 / 2-1 / 6$ and $1 / 6-1 / 10 \mu_{*}$ intervals, and $0.0-0.1,0.1-0.3$, $0.3-0.45$ and $0.45-1.0$ baryonic gas fraction $\left(f_{\text {gas }}\right)$ intervals. 

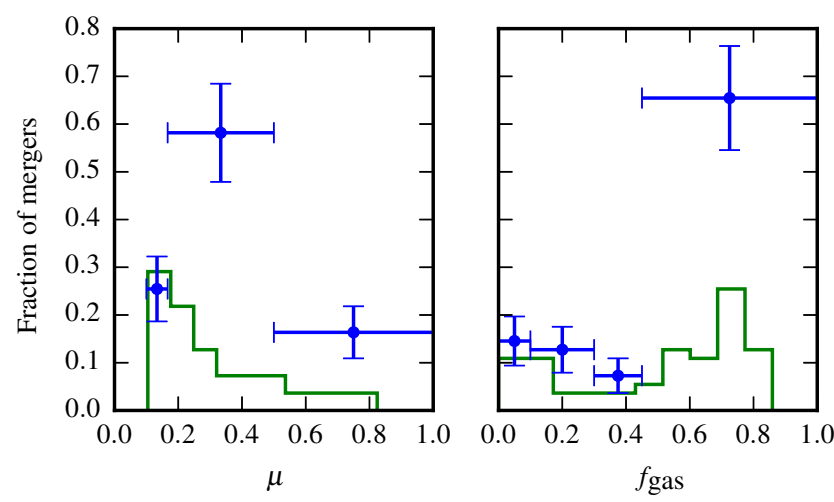

Figure 15. Normalized distribution of stellar mass ratios (left panel) and gas fractions (right panel) for mergers in the I-1 simulation at $z=0$ (green lines). Galaxies were selected with total stellar $\operatorname{mass}_{*}>10^{10} \mathrm{M}_{\odot}$ and stellar mass ratios $\mu_{*}>0.1$. For comparison, the estimations by using the intervals of Lotz et al. (2011) are included. Error bars in the y-axis denote Poisson noise in the number of mergers in a given interval, while error bars in the $\mathrm{x}$-axis denote bin widths.

Since for the $G-M_{20}$ criterium the individual observability time-scales $T_{i, j}$ are not a strong function of $f_{\text {gas }}$ (Lotz et al. 2010b), we average $T_{i, j}$ over $f_{\text {gas }}$ for each of the three mass bins. Then, we perform the sum in Eq.17 over the stellar mass ratios $(i)$. Merging the $f_{\text {gas }}$ bins also contributes to improve the statistics in $w_{i, j}$, otherwise some bins remain with a low number of mergers (less than 5 ).

We obtained a value of $\left\langle T_{\text {obs }}\right\rangle \sim 0.20 \mathrm{Gyr}$ for the I-1 simulation at $z=0$. Lotz et al. (2011) estimated the same cosmological average observability value using three different cosmological galaxy evolution models: 0.2 Gyr (Somerville et al. 2008), 0.21 Gyr (Croton et al. 2006) and 0.22 Gyr (Stewart et al. 2009). It is encouraging that similar $\left\langle T_{\text {obs }}\right\rangle$ are obtained by using different theoretical approaches: the semi-analytic approach (Croton et al. 2006; Somerville et al. 2008), halo abundance matching (Stewart et al. 2009) and N-body hydrodynamical cosmological simulations such as I-1.

\subsection{Intrinsic and morphological merger rates}

Taking advantage that the simulation provides us with the assembly history of galaxies via the merger trees, we can calculate the intrinsic merger rate as a function of minimal mass ratio $\mu_{\min }$ as

$R_{\text {merg }}^{\text {intr. }}\left(\mu_{\min }\right)=\frac{N\left(\mu_{\min }\right)}{T_{\max }}$,

where $T_{\max }=0.13 \mathrm{Gyr}$ is the elapsing time between the $z=0$ snapshot and the previous snapshot and $N\left(\mu_{\min }\right)$ is the number of galaxies having experience a merger with mass ratio larger than $\mu_{\text {min }}$.

Figure 16 shows the cumulative (with respect to mass ratio) intrinsic merger ratio for the $\mathrm{I}-1$ simulation at $z=$ 0 . From this figure a quick assessment of the merger rate is possible. For example, the intrinsic rate at $\mu_{*}>0.1$ is approximately $0.06 \mathrm{Gyr}^{-1}$, which implies that roughly one

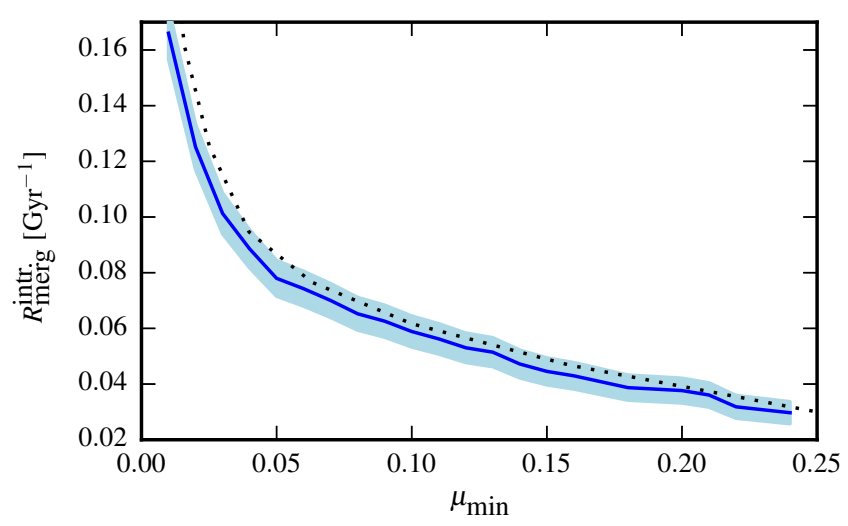

Figure 16. The cumulative intrinsic merger rate per galaxy for the I-1 simulation at $z=0$ (solid black line). The blue shaded region represents the Poisson noise. For the sake of comparison, the cumulative merger rate derived integrating the Rodriguez-Gomez et al. (2015) fitting function for the I-1 galaxygalaxy merger rate is included (dotted line).

in every 17 galaxies has experienced a $\mu_{*}>0.1$ merger in the last Gyr. Similarly, one in every 33 galaxies has experienced a major merger in the last Gyr.

We compare the intrinsic rate to the rate which would be derived using the non-parametric morphologies as proposed by Lotz et al. (2011)

$R_{\text {merg }}^{\text {morph. }}\left(\mu_{\min }\right)=0.5 \frac{N_{\text {above }}}{\left\langle T_{\text {obs }}\right\rangle}$,

where $N_{\text {above }}$ is the number of galaxies which are found above the LPM04 demarcation line. The value of 0.5 is the fiducial $C_{\text {merg }}$ that corresponds to the level of contamination derived in Section 4.3 According to the results of Section 5.1, we adopt $\left\langle T_{\text {obs }}\right\rangle=0.2 \mathrm{Gyr}$.

Therefore, by using our analysis of the I-1 simulation, we can compare the intrinsic merger rate with the morphologically derived one

$R_{\text {merg }}^{\text {intr. }}\left(\mu_{\min }\right)=C^{\prime} R_{\text {merg }}^{\text {morph. }}$.

The $C^{\prime}$ factor can be interpreted as a correction factor that brings the morphologically derived rate to the intrinsic merger rate of the simulation. If the computed $\left\langle T_{\text {obs }}\right\rangle$ is correct, that is, if the isolated merger observability time-scales $T_{i, j}$ are correct, then one would expect the $C^{\prime}$ factor to be close to unity.

Fig. 17 shows the correction factor $C^{\prime}$ as a function of $\mu_{\text {min }}$. We find that for a minimum mass ratio of $\sim 0.1$ no further correction factor is necessary to recover the intrinsic merger rate of the I-1 simulation. Lotz et al. (2011) found that the averaged observability time-scales derived from isolated pair simulations resulted in a global merger rate around a order of magnitude larger than predicted theoretical values (Somerville et al. 2008; Stewart et al. 2009; Croton et al. 2006; Hopkins et al. 2010b). These authors suggested that the discrepancy could be due to an overestimation of the $G-M_{20}$ merger rates because of a large contamination of non-merging system or to an underestimation of $\left\langle T_{\text {obs }}\right\rangle$. Alternatively, the theoretical models could be underestimating the frequency of minor mergers. Our re- 


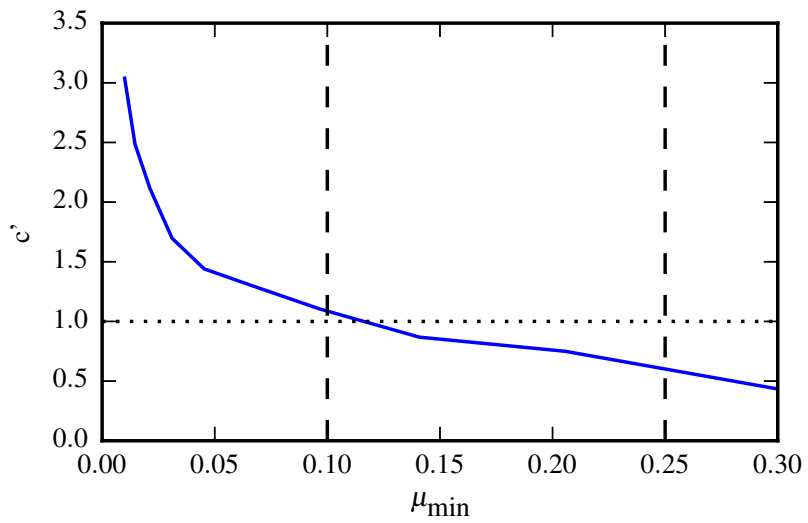

Figure 17. Correction fraction $C^{\prime}$ defined in equation 20 as a function of minimal mass ratio $\mu_{\min }$. The horizontal dotted line at $C^{\prime}=1$ intersects the curve at the mass ratio for which the morphologically derived rate recovers the intrinsic rate of I-1. As can be seen the intersection happens close to $\mu_{*}=0.1$, which is consistent with the ratio for which $G-M_{20}$ becomes sensitive to perturbations. The vertical dashed lines are shown for reference only; they denote the positions at $\mu_{*}=0.1$ and $\mu_{*}=0.25$

sults suggest that the $G-M_{20}$ method recovers the intrinsic merger fraction, favouring the idea that $\left\langle T_{\text {obs }}\right\rangle$ are well estimated using the described methods. Since $G-M_{20}$ morphologies are both sensitive to minor and major mass ratios. Figure 17 also suggests that a factor 0.625 correction can be used to determine the rate of exclusively major mergers from $G-M_{20}$ morphological studies.

In Figure 18 we show the major $\left(\mu_{*}>0.25\right)$ and total $\left(\mu_{*}>0.1\right)$ merger rates of I- 1 at $z=0$ as a function of descendant stellar mass. The blue dots represent the morphologically derived merger rate computed according to equation 19, but binning the merger candidates in stellar mass bins. As can be seen, the morphological derivation matches the intrinsic merger rate very well. This results further corroborates the estimated observability time-scales for the G$\mathrm{M}_{20}$ criteria; it is also compatible with results indicating that descendant mass does not affect $\mathrm{G}-\mathrm{M}_{20}$ observability time-scales (Lotz et al. 2011) which implies that no stellar mass bias is introduced in the merger rate derived using this method, at least in the $M_{*}>10^{10} \mathrm{M}_{\odot}$ range. Blue squares show the morphologically derived merger rate after applying the correction factor of 0.625 corresponding to major mergers, as can be seen, this correction results in a good match between the morphologically derived major merger rate as a function of descendant stellar mass and the intrinsic major rate. Finally, black triangles correspond to observations of the major merger rate by Casteels et al. (2014) derived using a similar morphological method as the one shown in the present work, but based on asymmetry and the merger time scales from Conselice (2006), rather than G-M $\mathrm{M}_{20}$. The Illustris simulation is in good agreement with this observations, as also noted by Rodriguez-Gomez et al. (2015).

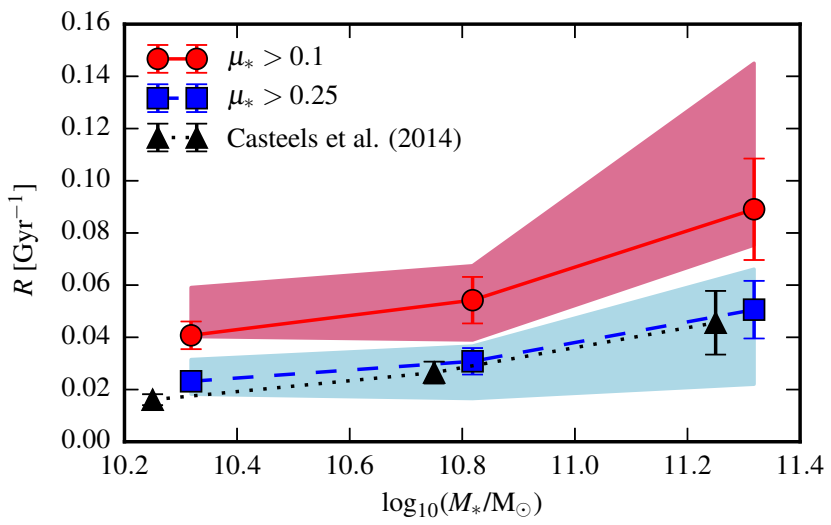

Figure 18. Galaxy merger rate as a function of descendant stellar mass estimated from our analysis of morphological $G-M_{20}$ for major mergers $\left(\mu_{*}>0.25\right.$, blue squares) and minor mergers $\left(\mu_{*}>0.1\right.$, red circles). Error bars show Poisson errors. The shaded regions represents intrinsic merger rate calculated from the I-1 simulation for the corresponding mass ratios. For the sake of comparison, the observational major merger rates derived using a morphological asymmetry method by Casteels et al. (2014) (black triangles).

\section{DISCUSSION AND CONCLUSIONS}

We studied non-parametric morphologies of $z=0$ simulated galaxy mergers in the cosmological context provided by the main Illustris simulation. From the publicly available idealized mock images, we produced mock g-band images comparable to the SDSS main galaxy survey. Then we characterized their morphologies by computing the following nonparametric morphology indicators: Gini, $M_{20}$, asymmetry, concentration and clumpiness for galaxies with $M_{*}>10^{10}$ $\mathrm{M}_{\odot}$. Our work allows us to bridge the gap between isolated merger simulations that explored a limited range of merger conditions and large scale simulations, which provide galaxy and merger properties and frequencies in agreement within the current cosmological paradigm.

We analysed the non-parametric morphologies of galaxies with recent mergers (within the last $2 \mathrm{Gyr}$ ) and with a close companion within $100 h^{-1} \mathrm{kpc}$. The non-parametric morphologies were correlated with the merger history and pair relative velocity and separation, respectively.

We also analysed the potential sources of contamination that affected a morphologically selected sample of merging galaxies in the Illustris simulation. We found that galaxies presenting an artificially generated ring-like structure constituted a large source of contamination, followed by lower mass irregular galaxies, with starbursting appearance. Edgeon galaxies and bursty spirals were other minor source of contamination. This is also commonly found in observational samples similarly selected. We were able to generate a clean sample of morphologically selected galaxies by removing these sources of contamination.

We found that $G-M_{20}$ morphologies of the complete galaxy sample reproduced well the trends previously reported by Snyder et al. (2015b) for individual subhalos of the Illustris simulation: bulge-dominated galaxies are lo- 
cated at high $G$, low $M_{20}$ values, while disc-dominated galaxies are found at low $G$, high $M_{20}$ values.

From the analysis of around $\sim 40000$ galaxies, we found close galaxies pairs $\left(d<20 h^{-1} \mathrm{kpc}\right)$ have a larger probability to be selected by $G-M_{20}$ morphologies ( $\sim 20$ per cent) and they are also well-selected by the asymmetry $A$. The analysis of the completeness shows that 50 per cent and 35 per cent of galaxies with positive merginess are in pairs with $d<45 h^{-1} \mathrm{kpc}$ and $\mu_{*}>0.25$ and $\mu_{*}>0.1$, respectively. Major merger remnants constituted the second subsample that the $G-M_{20}$ criteria was able to better differentiate, $\sim 5$ per cent of major merger remnants show perturbed morphologies. Nevertheless, $\sim 98$ per cent of the galaxies above the demarcation line have experienced a perturbation (i.e. a close interaction or a recent merger).

In agreement with previous works, the largest fraction of merger remnants and galaxy pairs are located below the demarcation line. However, the merginess is found to capture signatures of their actual state of disturbance. A clear correlation between the merginess and the elapsing time to the latest merger event is found. This trend is stronger for major merger events, and gets weaker when merger remnants of smaller mass ratios are included. However, all of them show merginess larger than the average of the whole sample.

Using the observability time-scales from isolated merger simulations by Lotz et al. (2008a, 2010b,b) and the mass ratios and gas fractions distribution of $z=0$ mergers in the Illustris main simulation, we computed the average observability time-scale of the simulation. We found a value of $\left\langle T_{\text {obs }}\right\rangle \sim 0.2 \mathrm{Gyr}$, very similar to other reported values from simulations and semi- analytical models (Lotz et al. 2011). Next, we put this value to the test, comparing the intrinsic merger rate of Illustris to the merger rate that would have been derived from morphological studies using the $G-M_{20}$ criterium. We found that after accounting for the contamination of morphologically selected galaxies, no further corrections where necessary to reconcile the intrinsic merger rate to the morphologically derived one. This agreement indicates that the computed average observability time-scales are a correct estimation of the time that $z=0$ mergers are detected above the demarcation line. This result validates the findings obtained by the isolated merger simulations and shows that the cosmological context of galaxy formation does not introduce effects that greatly alter the observability time-scales of merger events at least in the local Universe.

We notice that the discrepancy found by Lotz et al. (2011) between the observed total merger rate (minor plus major mergers) and the intrinsic merger rate from simulations (Hopkins et al. 2010a; Somerville et al. 2008; Stewart et al. 2009) is maintained in the Illustris simulation. Observational estimations indicate a large merger rate (0.37 $\mathrm{Gyr}^{-1}$ at $\left.z=0.3\right)$, while Illustris predicts a much lower rate $\left(0.06 \mathrm{Gyr}^{-1}\right.$ at $\left.z=0\right)$. This large difference can not be explained by reasonable evolutionary trends of the merger rate with redshift. Lotz et al. (2011) proposed several possible solutions to this discrepancy: simulations could be under-predicting the merger rate of minor mergers, the observability time- scales of $G-M_{20}$ morphologies could be underestimated or observations could be affected by large contamination from non-merging systems. We have shown that computing average observability time-scales using the standard procedure results in correctly derived observed merger rates, compatible to the intrinsic merger rate. We also note that, completely disregarding contamination sources in our estimations of the morphological derived rates produces a merger rate of $0.2 \mathrm{Gyr}^{-1}$, closer to the reported observational values. Our results suggest that contamination sources might explain this discrepancy, which seems to be more likely associated to minor merger events. Indeed, major merger rates are well reproduce by the I- 1 simulation when compared to non-parametric morphological studies using the asymmetry statistic (Casteels et al. 2014) and we have shown that $G-M_{20}$ morphologies can reproduce the major merger rate if a correction factor of $\sim 0.63$ is applied.

We also studied the effects of mergers on the asymmetry statistics $A$. We found that asymmetry increased for close pair with $d<35 h^{-1} \mathrm{kpc}$. However we found that asymmetry greatly increases for lower mass galaxies, resulting in most galaxies with $M_{*}<10^{10.5} \mathrm{M}_{\odot}$ with $A>0.35$, regardless of the presence or absence of any merging event. Based on previous works, we suggest that this effect might be largely caused by the combination of low numerical resolution for low mass galaxies and stochastic formation of stellar particles that greatly affect the appearance of galaxies. Although this renders the asymmetry statistic of the simulation very hard to properly compare with observations, as this effect is intrinsic to simulations, we found the trend with $A$ is present at all mass intervals. The larger changes of $A$ is detected for higher stellar mass galaxies in pairs with $d<30$ $h^{-1} \mathrm{kpc}$. Smaller galaxies show an increasing level of $A$ at all relative distances which remains to be confirmed with higher numerical resolution simulations. We also analysed the dependence of $A$ with relative velocities, galaxies with $V<\sim 300 \mathrm{~km} \mathrm{~s}^{-1}$ have $A>0.35$, while pairs with higher relative velocities have $A<0.35$, suggesting that flyby events have no significant impact on morphological disturbances.

\section{ACKNOWLEDGEMENTS}

The authors acknowledge the grants PICT 2011-0959 from Argentinian ANPCyT, and PIP 2012-0396 from Argentinian CONICET and Fondecyt Regular 115033, Southern Astrophysics Network Redes Conicyt 150078 and proyecto interno MUN UNAB 2015. This research made use of Astropy (Robitaille et al. 2013), numpy (Walt et al. 2011), and matplotlib (Hunter 2007)

\section{REFERENCES}

Abraham R. G., Tanvir N. R., Santiago B. X., Ellis R. S., Glazebrook K., Bergh S. v. d., 1996, MNRAS, 279, L47

Barton E. J., Geller M. J., Kenyon S. J., 2000, ApJ, 530, 660

Bell E. F., et al., 2008, ApJ, 680, 295

Bershady M. A., Jangren A., Conselice C. J., 2000, Aj, 119, 2645

Borne K. D., Bushouse H., Lucas R. A., Colina L., 2000, ApJ, 529, L77

Brinchmann J., et al., 1998, ApJ, 499, 112

Bundy K., Ellis R. S., Conselice C. J., 2005, ApJ, 625, 621

Bundy K., Fukugita M., Ellis R. S., Targett T. A., Belli S., Kodama T., 2009, ApJ, 697, 1369

Casteels K. R. V., et al., 2014, MNRAS, 445, 1157 
Charlot S., Fall S. M., 2000, ApJ, 539, 718

Conselice C. J., 2003, ApJS, 147, 1

Conselice C. J., 2006, ApJ, 638

Conselice C. J., Bershady M. A., Jangren A., 2000, ApJ, 529, 886

Conselice C. J., Bershady M. A., Dickinson M., Papovich C., 2003, $\mathrm{Aj}, 126,1183$

Conselice C. J., Yang C., Bluck A. F. L., 2009, MNRAS, 394, 1956

Croton D. J., et al., 2006, MNRAS, 365

Davis M., Efstathiou G., Frenk C. S., White S. D. M., 1985, ApJ, 292, 371

De Propris R., Liske J., Driver S. P., Allen P. D., Cross N. J. G., 2005, $\mathrm{Aj}, 130,1516$

Di Matteo P., Combes F., Melchior A.-L., Semelin B., 2007, A\&A, 468, 61

Dolag K., Borgani S., Murante G., Springel V., 2009, MNRAS, 399, 497

Ellison S. L., Patton D. R., Simard L., McConnachie A. W., 2008, $\mathrm{Aj}, 135,1877$

Ellison S. L., Mendel J. T., Patton D. R., Scudder J. M., 2013, MNRAS, 435, 3627

Genel S., Genzel R., Bouché N., Naab T., Sternberg A., 2009, ApJ, 701, 2002

Genel S., et al., 2014, MNRAS, 445, 175

Guo Q., White S. D. M., 2008, MNRAS, 384, 2

Hinshaw G., et al., 2013, ApJS, 208, 19

Hopkins P. F., Hernquist L., Cox T. J., Di Matteo T., Robertson B., Springel V., 2006, ApJS, 163, 1

Hopkins P. F., et al., 2010a, ApJ, 715, 202

Hopkins P. F., et al., 2010b, ApJ, 724, 915

Huertas-Company M., et al., 2014, Measuring galaxy morphology at $\$ \mathrm{z}>1 \$$. I - calibration of automated proxies, http://adsabs.org/2014arXiv1406.1175H

Hunter J. D., 2007, Computing in Science Engineering, 9, 90

Isserstedt J., Schindler R., 1986, A\&A, 167

Johnston K. V., Hernquist L., Bolte M., 1996, ApJ, 465, 278

Jonsson P., 2006, MNRAS, 372, 2

Jonsson P., Groves B. A., Cox T. J., 2010, MNRAS, 403, 17

Kampczyk P., et al., 2007, ApJS, 172, 329

Lambas D. G., Tissera P. B., Alonso M. S., Coldwell G., 2003, MNRAS, 346, 1189

Leitherer C., et al., 1999, ApJS, 123, 3

Leitherer C., Ortiz Otálvaro P. A., Bresolin F., Kudritzki R.-P., Lo Faro B., Pauldrach A. W. A., Pettini M., Rix S. A., 2010, ApJS, 189, 309

Lin L., et al., 2004, ApJ, 617, L9

López-Sanjuan C., Balcells M., Pérez-González P. G., Barro G., García-Dabó C. E., Gallego J., Zamorano J., 2009, A\&A, 501, 505

Lotz J. M., Primack J., Madau P., 2004, Aj, 128, 163

Lotz J. M., Jonsson P., Cox T. J., Primack J. R., 2008a, MNRAS, 391,1137

Lotz J. M., et al., 2008b, ApJ, 672, 177

Lotz J. M., Jonsson P., Cox T. J., Primack J. R., 2010a, MNRAS, 404,575

Lotz J. M., Jonsson P., Cox T. J., Primack J. R., 2010b, MNRAS, 404,590

Lotz J. M., Jonsson P., Cox T. J., Croton D., Primack J. R., Somerville R. S., Stewart K., 2011, ApJ, 742, 103

Mihos J. C., Hernquist L., 1994, ApJ Letters, 437, L47

Mihos J. C., Hernquist L., 1996, ApJ, 464, 641

Naab T., Burkert A., 2003, ApJ, 597, 893

Patton D. R., et al., 2002, ApJ, 565, 208

Perez M. J., Tissera P. B., Scannapieco C., Lambas D. G., Rossi D., E M., 2006, A\&A, 459

Robertson B., Bullock J. S., Cox T. J., Di Matteo T., Hernquist L., Springel V., Yoshida N., 2006, ApJ, 645, 986

Robitaille T. P., et al., 2013, A\&A, 558, A33
Rodriguez-Gomez V., et al., 2015, MNRAS, 449, 49

Scudder J. M., Ellison S. L., Torrey P., Patton D. R., Mendel J. T., 2012, MNRAS, 426, 549

Sijacki D., Springel V., Di Matteo T., Hernquist L., 2007, MNRAS, 380

Sijacki D., Vogelsberger M., Genel S., Springel V., Torrey P., Snyder G. F., Nelson D., Hernquist L., 2015, MNRAS, 452, 575

Snyder G. F., Lotz J., Moody C., Peth M., Freeman P., Ceverino D., Primack J., Dekel A., 2015a, MNRAS, 451, 4290

Snyder G. F., et al., 2015b, MNRAS, 454, 1886

Somerville R. S., Primack J. R., Faber S. M., 2001, MNRAS, 320, 504

Somerville R. S., Hopkins P. F., Cox T. J., Robertson B. E., Hernquist L., 2008, MNRAS, 391, 481

Sparre M., Springel V., 2016, preprint, 1604, arXiv:1604.08205

Sparre M., et al., 2015, MNRAS, 447, 3548

Springel V., 2010, MNRAS, 401, 791

Springel V., White S. D. M., Tormen G., Kauffmann G., 2001, MNRAS, 328, 726

Stewart K. R., Bullock J. S., Barton E. J., Wechsler R. H., 2009, ApJ, 702, 1005

Takamiya M., 1999, ApJS, 122, 109

Torrey P., et al., 2015, MNRAS, 447, 2753

Trayford J. W., et al., 2015, MNRAS, 452, 2879

Vogelsberger M., Genel S., Sijacki D., Torrey P., Springel V., Hernquist L., 2013, MNRAS, 436, 3031

Vogelsberger M., et al., 2014, MNRAS, 444, 1518

Walt S. v. d., Colbert S. C., Varoquaux G., 2011, Computing in Science \& Engineering, 13, 22

White S. D. M., Rees M. J., 1978, MNRAS, 183, 341

Wu K. L.-K., 1999, Ph.D. Thesis

de Ravel L., et al., 2009, A\&A, 498, 379

\section{APPENDIX A: NUMERICAL APPROACH: COMPARISON TO DIGGSS MORPHOLOGIES}

"Dusty Interacting Galaxy GADGET-SUNRISE Simulations" (DIGGSS) ${ }^{2}$ are series of isolated merger simulations used to derived the non-parametric merger time-scales in Lotz et al. (2008a, 2010b,a). In order to validate and calibrate our approach to the computation of non-parametric morphologies, we recalculated those of the DIGGSS mock images and compared them to the tabulated results.

Figure A1 compares our results for the Gini and $M_{20}$ statistics to the tabulated values for the g3iso galaxy, whose characteristic are listed in table A1. Each point in the figure represents a mock image of the galaxy taken at a certain time in the simulation, with one of the eleven cameras distributed around the galaxy. The lower panels represent the relative deviation from the tabulated value. As can be seen a good general agreement was found, most of our estimations lie within 10 per cent of the tabulated value, with a few outliers for the Gini statistic at low $G$, and for $M_{20}$ at low and high values.

Similarly, Fig A2 compares our results for the concentration and asymmetry. The Concentration presents a slight bias towards lower concentration values that increases towards higher concentrations, but all results are within 10 per cent of the tabulated values. The asymmetry agrees well with the tabulated values specially at higher asymmetry values. For low asymmetry there is larger dispersion.

2 http://archive.stsci.edu/prepds/diggss 
Table A1. DIGGSS g3iso fundamental parameters.

\begin{tabular}{ll}
\hline Virial mass & $1.2 \times 10^{12} \mathrm{M}_{\odot}$ \\
Dark matter halo concentration & 6 \\
Baryonic mass & $6.2 \times 10^{10} \mathrm{M}_{\odot}$ \\
Mass of stellar disc & $4.1 \times 10^{10} \mathrm{M}_{\odot}$ \\
Mass of stellar bulge & $8.9 \times 10^{10} \mathrm{M}_{\odot}$ \\
Mass of gaseous disc & $1.2 \times 10^{10} \mathrm{M}_{\odot}$ \\
Fraction of baryons in the bulge & 0.14 \\
Fraction of baryons in gas & 0.19 \\
Scalelength of stellar disc & $2.85 \mathrm{kpc}$ \\
Scalelength of bulge & $0.62 \mathrm{kpc}$ \\
Scalelength of gaseous disc & $8.55 \mathrm{kpc}$ \\
\hline
\end{tabular}

These results show that our approach is able to produce robust non-parametric morphologies comparable to the ones derived in previous works.

This paper has been typeset from a $\mathrm{T}_{\mathrm{E}} \mathrm{X} / \mathrm{LAT}_{\mathrm{E}} \mathrm{X}$ file prepared by the author. 

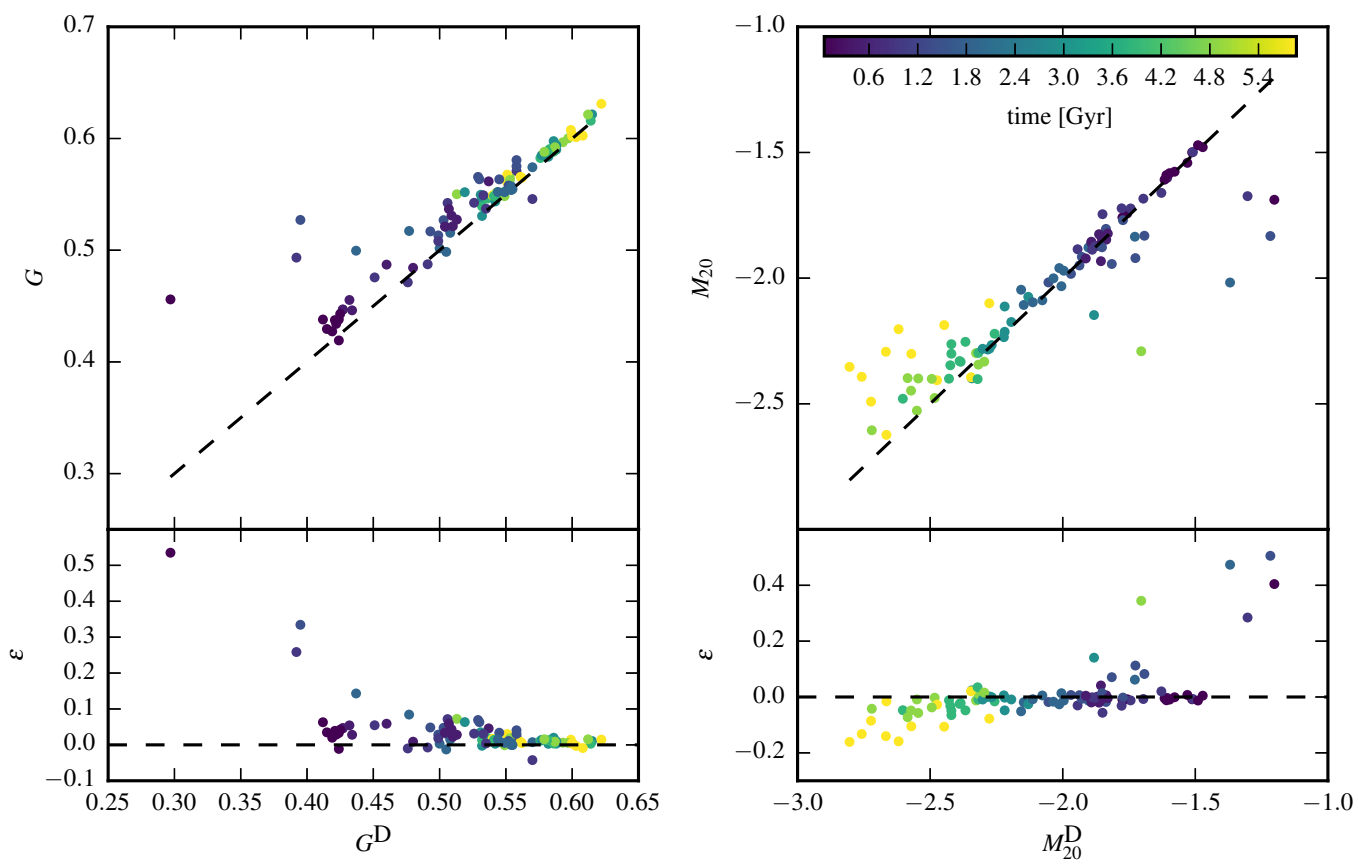

Figure A1. Left: gini computed using the methods described in this paper vs. tabulated values for the DIGGSS g3iso galaxy. Right: the same for the $M_{20}$ statistic. Colours represent the time since the start of the simulation. In both cases, lower panels represent the relative deviation of the parameters computed using our method to the tabulated values.
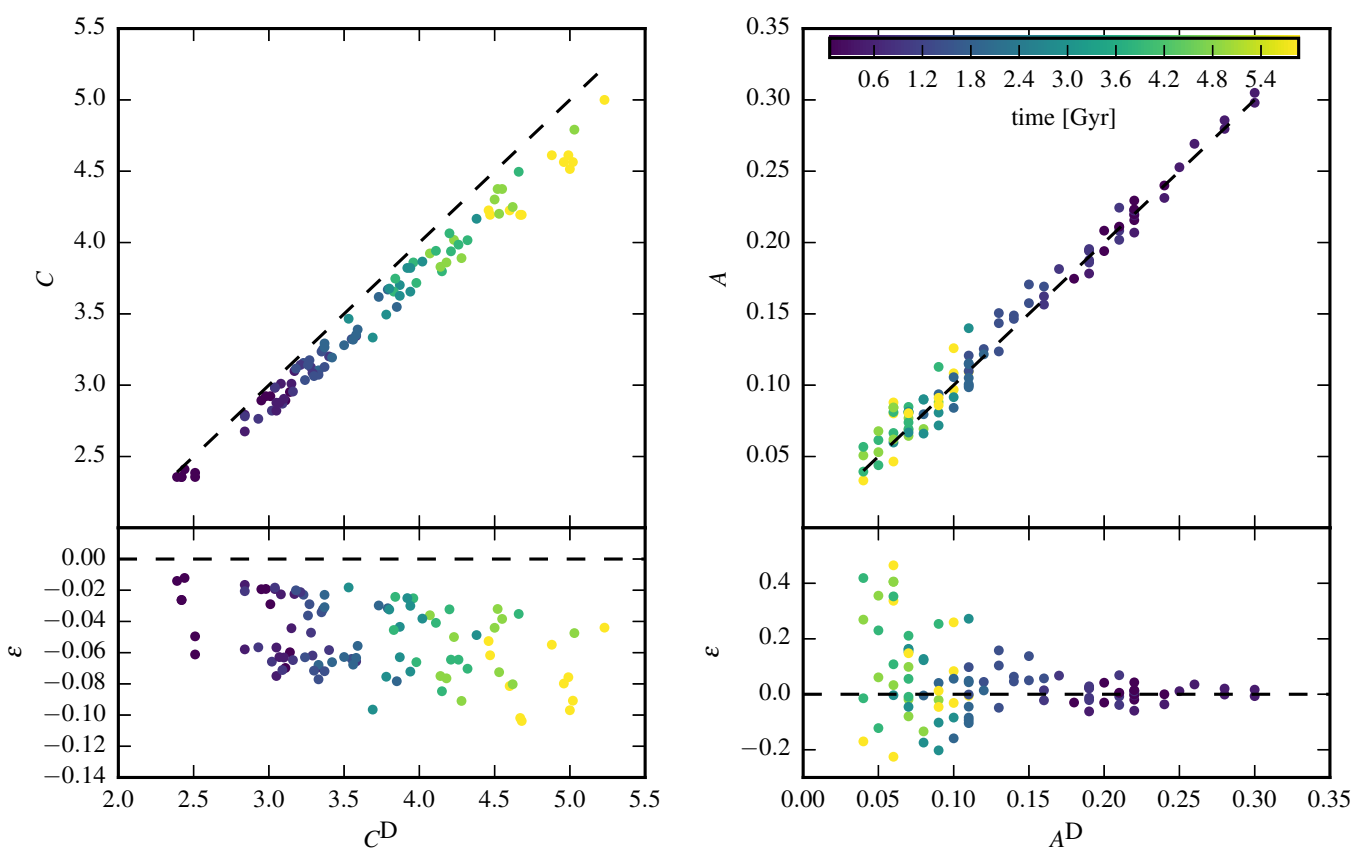

Figure A2. Left: concentration computed using the methods described in this paper vs. tabulated values for the DIGGSS g3iso galaxy. Right: the same for the asymmetry statistic. Colours represent the time since the start of the simulation. In both cases, lower panels represent the relative deviation of the parameters computed using our method to the tabulated values 